\title{
LES SENS DE “SUBSTANCE” CHEZ APOLLONIUS DYSCOLE
}

\author{
JULIE BRUMBERG-CHAUMONT \\ Centre National de la Recherche Scientifique (France)
}

\begin{abstract}
RÉSUMÉ: Larticle consacre un examen détaillé aux sens de "substance" présents chez Apollonius Dyscole. La première difficulté est d'ordre méthodologique, puisqu'il faut s'interroger sur une notion éminemment philosophique au sein d'un texte grammatical de l'Antiquité tardive. Il faut donc préciser quel peut bien être le référent philosophique auquel peut être rapporté l'usage apollonien de la notion de substance, en tenant compte de la forte présence de philosophèmes stoïciens et aussi d'une variété d'influences péripatéticiennes, médioet néo-platoniciennes. À cette difficulté méthodologique s'ajoute l'obstacle d'un corpus lacunaire, auquel il manque la partie sur le nom. Il faut donc reconstituer la doctrine apollonienne sur la base de ce qu'on trouve, de manière assez éparse, dans la Syntaxe et dans le Peri antônumias. Alors plusieurs éléments mettent en question l'adhésion d'Apollonius à la formule du Peri antônumias, laquelle ne correspond pas aux formules habituelles de la Syntaxe, ni au cœur de sa doctrine sur le rapport de complémentarité entre nom et pronom au "Livre II", qui est censé être postérieur au Peri antônumias et constituer une synthèse de l'enseignement du grammairien grec. Apollonius manierait alors de manière implicite non seulement les deux sens de "substance" comme qualité substantielle et comme propriété de signification attachée en propre aux pronoms, mais aussi un troisième sens de "substance", la substance-substrat.
\end{abstract}

MOTS-CLEFS: Apollonius Dyscole, Priscien; ousia, huparxis, hupokeimenon, deixis; substantia, subjectum; substance, qualité, quantité; nom, adjectif, pronom.

\section{Introduction: trois sens de "substance" chez Apollonius comme chez Priscien?}

L'enquête que nous proposons sur les différents sens de "substance" chez Apollonius fait suite à des travaux antérieurs sur les sens de "substance" chez l'héritier 
direct du grammairien alexandrin dans le monde latin, Priscien. Confronté aux incohérences apparentes de Priscien, qui affirme tantôt que tous les noms signifient la substance et la qualité - c'est là le propre du nom - tantôt que certains noms seulement signifient la substance, d'autres la qualité, d'autres, enfin, la quantité, M. Baratin a proposé de distinguer deux sens de "substance" et de "qualité". Il s'agit d'une part de la substance-sujet, signifiée par tous les noms, et par les pronoms (qui signifient la seule substance), et, d'autre part, de la substance comme "qualité substantielle" signifiée seulement par certains noms, comme "homme", "animal", ceux qui correspondent aux noms dans la catégorie de la substance chez Aristote. Quant à la qualité, elle désigne ou bien la détermination avec laquelle la substance est désignée, ce qui s'applique à tous les noms, ou bien la qualité au sens strict, au sens des Catégories (par opposition à la substance ou la quantité). Elle est alors signifiée par les seuls adjectifs "qualificatifs", tel que "blanc". Ainsi, tout nom signifie la substance et la qualité chez Priscien, que M. Baratin désigne respectivement par substance 1 et qualité 1 . La substance et la qualité peuvent par ailleurs être les deux premiers éléments d'une série déterminant le deuxième élément du propre, la qualité 1: cette qualité peut être soit une substance 2 (comme pour "animal", "homme"), soit une qualité 2 (comme pour "blanc", "noir"), soit une quantité ("de-deux-pieds") (Baratin, 1989: p. 400-402).

Dans une étude précédente, nous avons tenté de montrer qu'il était opportun de distinguer non pas seulement deux sens de "substance" chez Priscien, mais bien au moins trois sens de "substance". Outre la substance comme "qualité substantielle" et la substance signifiée par tous les noms comme substrat des propriétés, deux signifiés du nom, il convient de distinguer encore la substance signifiée par les pronoms, laquelle est une propriété de signification (Lallot, 1988: p. 18-19) - un mode de signifier et pas seulement un signifié. Nous avons comparé cette interprétation aux sens de "substance" que Pierre Hélie (XIIe siècle) avait proposés à propos de la signification du nom propre, du nom appellatif et du pronom, en se fondant sur les sens de "substance" distingués au Moyen Âge dans les commentaires aux traités théologiques de Boèce (Brumberg-Chaumont, 2009).

Il est vrai que plusieurs éléments pourraient laisser penser que la distinction de ces différents sens de "substance" n'est exigée en propre que par l'adaptation que Priscien fait de la grammaire d'Apollonius, et qu'elle ne concerne pas cette dernière directement, prise en elle-même. D'abord, la distinction des trois sens de "substance" proposée par Pierre Hélie est partiellement due à un problème de traduction du grec au latin pour un passage crucial de la Syntaxe: substantia vient traduire à la fois ousia 
et huparxis, rendant le passage incompréhensible faute de distinguer plusieurs sens de substantia 1 . Le maniement de plusieurs sens de "substance" dans les Institutions Grammaticales est en outre lié à la définition du propre du nom formulée par Priscien (GL II, p. 55, 6: "le propre du nom est de signifier la substance et la qualité"): c'est en raison de cette formule qu'il a fallu distinguer la substance signifiée par tous les noms (la substance-substrat des qualités) et la substance comme détermination substantielle, qui n'est signifiée que par les noms de substances comme "homme". Or on ne peut attribuer avec autant de certitude cette description de la propriété de signification du nom à Apollonius, en raison de la perte de la plupart de ses ouvrages sur les parties du discours. On trouve bien la formule "le nom [signifie] la substance avec la qualité" (voir infra § 5) dans le Peri antônumias d'Apollonius passage effectivement cité en parallèle avec la formule de Priscien sur le propre du nom par l'éditeur des fragments des œuvres perdues d'Apollonius (GG II/3, p. 38, 1 . 20) et considéré unanimement par les spécialistes comme un texte ayant un statut théorique comparable à celui de Priscien. Quant à la définition que Priscien donne du nom², qui comporte deux corrélats sémantiques, la qualité distribuée et les "sujets" (hupokeimena, subjecta) auxquels cette qualité est distribuée, elle a été attribuée à Apollonius lui-même dans les Scholies à la Technè (voir infra Texte 11). De là à penser que les "sujets" de la définition attribuée par les Scholies à Apollonius et la "substance" mentionnée par le Peri antônumias sont équivalents, comme ils le seraient chez Priscien, il n'y a qu'un pas, généralement accompli par les commentateurs, anciens ou modernes. Mais la situation n'est pas si claire: d'après ce qu'on peut reconstituer de la Syntaxe, de nombreux passages nous indiquent que le nom signifie la qualité, tandis que le pronom signifie la substance ${ }^{3}$, ce qui explique leur complémentarité,

1 Il s'agit du passage de la Syntaxe cité infra à la note 39, et repris dans le Texte 2, au $§ 3$. Comme on le voit dans le texte plus complet cité en note 31, l'utilisation de substantia pour la traduction d'huparxis est certes imprécis, mais pas complètement dénué de fondement, dans la mesure où le pronom qui interroge sur l'huparxis, l'"identité", ou l'“existence”, selon les traductions habituellement retenues pour ce vocable chez Apollonius, est en même temps celui qui n'interroge que sur l'ousia, la substance, dans la suite immédiate du texte.

2 "Le nom est une partie du discours qui assigne une qualité, commune ou particulière, à chacun des sujets, corps ou choses" (GL II, p. 56, 27-57, 1).

3 "En ce qui concerne Apollonius, il est exact qu'il définit ordinairement le nom (et l'appellatif) en termes de poiotès ... l'ousía étant pour lui le signifié propre (idía énnoia) du pronom", J. Lallot, La Grammaire de Denys le Thrace, Paris, 1998 (2 ${ }^{\text {nde }}$ édition), p. 128 (notes). Nous soulignons. Immédiatement à la suite, J. Lallot signale a contrario le texte du Peri antônumias que nous avons évoqué. 
même à la troisième personne où noms et pronoms sont possibles. D'autre part, le statut théorique du passage du Peri antônumias cité précédemment nous semble sujet à caution (voir infra § 5). Il est par conséquent pertinent de consacrer un examen détaillé aux sens de "substance" présents chez Apollonius Dyscole de la même façon qu'on s'est penché sur cette question chez Priscien, selon une suggestion de M. Baratin lui-même dans son étude sur le grammairien latin, qui voit chez Apollonius les prémices de la double acception de "substance" et de "qualité" telle qu'on la trouve développée par Priscien (voir infra n. 22).

\section{Les interactions entre grammaire et philosophie dans l'Antiquité tardive: pro- blèmes de méthode}

Avant d'entrer dans le détail de l'argumentation, quelques considérations méthodologiques s'imposent pour une étude qui s'interroge sur une notion éminemment philosophique, celle de substance, au sein d'un texte grammatical de l'Antiquité tardive. Il ne va en effet pas de soi que "substance" ait un "sens", et encore moins un "sens philosophique". Il faut préciser le contenu qu'on donne à une telle enquête, et quel peut bien être le référent philosophique auquel on entend rapporter l'usage apollonien de la notion de substance. La formulation même de la question suggère la difficulté de la tâche, au regard du syncrétisme qui marque la philosophie de l'Antiquité tardive en général, et ce type de notions en particulier.

C'est bien un référent philosophique complexe qu'il convient de mobiliser quand on s'intéresse aux relations entre grammaire technique et philosophie dans l'Antiquité tardive. A. Luhtala ${ }^{4}$ a souligné comment l'adoption désormais généralisée d'une datation tardive de la Technè grammatikè attribuée à Denys le Thrace, du moins pour sa "partie systématique" (chapitres 6 à 20, ou chapitres 11 à 20)5 changeait la donne. Il n'y a pas eu simplement un "relais" (Blank, 1982: p. 4) des préoccupations linguistiques, de la philosophie à une grammaire en voie de technicisation, durant la période hellénistique, mais bien des interactions constantes entre philosophie et grammaire durant la période impériale et l'Antiquité tardive, jusqu'à la canonisation

4 Dans l'introduction de son ouvrage Grammar and Philosophy in Late Antiquity (Amsterdam, 2005).

5 Sur cette question, voir le résumé des débats fourni par Lallot (1998: p. 20-26) et Ildefonse (1997: p. 448 sqq): la Technè serait le fruit d'un remaniement par un scholiaste tardif des Préceptes de Denys le Thrace à partir de l'enseignement d'Apollonius et d'Hérodien. 
de la grammaire technique issue d'Apollonius et d'Hérodien (aux III- IVe siècles) dont la deuxième partie de la Technè est précisément une réalisation majeure - et au-delà. Outre la forte présence de philosophèmes stoïciens, reconnue par tous, il faut donc prendre en compte l'existence d'une variété d'influences philosophiques, où, le médio-platonisme ${ }^{6}$, le péripatétisme, puis après Apollonius, Porphyre, et, d'une manière plus générale, le néo-platonisme, ont leur part. Cet ensemble est désigné par A. Luhtala par l'expression "hellenistic syncretism" (p. 30-37), tandis que S. Ebbesen a récemment proposé le signe "logical LAS", pour "Logical Late Ancient Standard", qui fait pendant au "grammatical LAS" (Ebbesen, 2007), J. Lallot évoquant la "position fondamentalement syncrétique de l'école grammaticale alexandrine" (Lallot, 1998: p. 38-39).

Cette approche implique notamment de prendre considération l'idée que les éléments doctrinaux de la période hellénistique (surtout stoïciens) recyclés par Apollonius, puis par la tradition apollonienne (Lallot, 2004), sont déjà "contaminés" par des interprétations plus tardives, notamment péripatéticien. Certaines doctrines un temps attribuées aux Stoïciens anciens sur la base de rares témoignages tardoantiques, ont été finalement rapportées à une interprétation tardive du Stoïcisme, ou restituées à l'adaptation que Porphyre avait faite de ces éléments. C'est en particulier le cas de la distinction entre qualité propre et qualité commune au sein des catégories stoïciennes ${ }^{7}$, de la correspondance entre catégories stoïciennes et

6 Sur le débat entre philosophie et grammaire au sein de cette tradition, voir Wouters (1996).

7 On a associé des témoignages sur les catégories stoïciennes, qui mentionnent seulement le "qualifié", au témoignage de Diogène Laërce sur les qualités, communes et particulières, signifiées respectivement par l'appellation et par le nom, ce qui a justement permis de proposer un parallélisme entre catégories, signifiés et expressions . Celle-ci a été analysée par l'érudition moderne comme un "montage" tardif qui détourne l'attention du rôle que doit jouer le "qualifié particulièrement" et la distinction des deux premières catégories dans la métaphysique et la physique stoïcienne pour répondre à l' "argument croissant" (voir Long; Sedley, 2001: p. 24; infra n. 11). Il est vrai que nous disposons d'une source (Simplicius, In Categorias, CAG VIII, p. 48, 11-15 = Fr. 55 Smith) qui mentionne l'existence chez les Stoïciens, suivant Porphyre, des deux sujets (la matière première, premier signifié de "sujet", et, secondairement, le sujet qui subsiste "communément ou particulièrement qualifié (ho koinôs poion è idiôs huphistatai)"), ce qui correspondrait à la distinction des deux premières catégories associée à la distinction entre la qualité commune et la qualité particulière. Mais il s'agit d'un extrait du commentaire de Porphyre sur les Catégories "ad Gedalios", qui n'est probablement pas fidèle à la doctrine stoïcienne; il a d'ailleurs été récemment analysé par R. Chiaradonna comme un exemple typique où des notions stoïciennes et aristotéliciennes se contaminent: "Porphyry, then, 
parties du discours (voir infra n. 11), et de la définition de la qualité particulière à partir d'une collection unique de propriétés ${ }^{8}$ - trois déplacements textuels et doctrinaux sous influence aristotélicienne qui nous portent au cœur de la question de la substance et à de son usage en grammaire.

Ce cadre de réflexion est particulièrement pertinent pour la question de la "substance", notion probablement ni exclusivement aristotélicienne, stoïcienne ou platonicienne, dans les nombreux contextes où elle est opératoire en cette fin d'Antiquité, en philosophie comme en grammaire'. Le double sens de l'ousia, entre substrat et essence, le recouvrement de la distinction entre substance première et substance seconde par celle entre sujet qualifié particulièrement et communément, la fusion entre la qualité particulière stoïcienne et la collection de propriétés porphyrienne, le maniement complexe de la notion de "qualité substantielle", ou encore l'idée de substance seconde comme "qualité" dans les Catégories d'Aristote ${ }^{10}$,

fitted Aristotelian and Stoic notions about matter, qualities and 'subjects' within a single doctrinal framework. Not only did he adopt current terms of Stoic origin, terms that by Porphyry's day were bereft of distinctive philosophical connotations; but, at least in some cases, he also consciously made use of Stoic theories in his exegesis of Aristotle's Categories (cf. Simpl., In Cat., 2, 8). This passage [i. e. Fr. 55 Smith] suggests that Porphyry integrated Aristotle's theory of substance and the Stoic theory of matter and quality the individual substance of Aristotle's Categories being conceived, then, as 'what subsists [...] peculiarly qualified"” (Chiaradonna, 2008: p. 15).

8 Comme l'a montré R. Chiaradonna, c'est une notion proprement porphyrienne, qui n'a été rapportée que tardivement au Stoïcisme par les sources anciennes, suivies en cela par de certains érudits modernes, à partir du témoignage de Dexippe (In Categorias, CAG IV/2, éd. A. Busse, Berlin, G. Reimer, 1888, p. 30, 23-26) (Chiaradonna, 2000: p. 119).

9 Voir notamment la remarque de F. Ildefonse (1997: p. 262), qui mentionne aussi le registre de la signification (1997: p. 290-292).

10 À propos de la "qualité substantielle", les exégètes néoplatoniciens ont débattu du statut ontologique de la différence spécifique, pour savoir si ce "complément de substance" était lui-même une substance, ou une qualité, ou un intermédiaire entre les deux, une qualité substantielle, voir notamment Dexippe (In Categorias, CAG IV/2, p. 49, 4-25), Simplicius (In Categorias, CAG VIII, éd. C. Kalbfleisch, Berlin, G. Reimer, 1907, p. 98, 1-35) et Boèce (In Categorias, PL VXIV, 192 A-C), ainsi que la synthèse de C. Luna (2001: p. 242-243). Outre la question du statut ontologique particulier de la différence, ce débat a pour fondement la description des substances secondes comme des qualités, substantielles en Catégories 1b 10-24, où la substance seconde est décrite comme un qualifié (poion ti) relativement à la substance. On trouve là une source philosophique importante à l'idée qu'il existe plusieurs sens de "substance" et de "qualité" de sorte que certaines qualités sont en réalité des substances (et inversement) ce qui est précisément le cas de la qualité au sens 1 lorsqu'elle est une substance au sens 2 chez Priscien. Il y aurait de nombreux textes à citer, mais on peut notamment se référer à Porphyre 
tous ces éléments constitutifs de ce que S. Ebbesen qualifie de LAS (Late Ancient Standard) en logique et en philosophie, forment un fond disponible pour de nouvelles élaborations théoriques au sein de la réflexion linguistique de l'Antiquité tardive.

À cette fluidité du référent philosophique s'ajoute l'écart des usages, entre une enquête sur les choses et sur les mots. Si les notions grammaticales de substance et de qualité ne sont pas moins ultimement ancrées dans une description physique des déterminations qui s'agrègent à un sujet de base que ne le sont les catégories stoïciennes, les spécialistes de la logique et de la grammaire anciennes ont souligné depuis quelques décennies combien il convenait de se garder de voir une correspondance terme à terme entre catégories "ontologiques", et parties du discours grammaticales. Comme nous l'avons vu à propos des qualités particulières et communes, il convient de s'en garder dans le cadre du Stoïcisme ancien puisqu'il est apparu que le tableau qu'on avait dressé de ces correspondances s'avère être une reconstruction factice, ou du moins un écho très amoindri de la doctrine stoïcienne ancienne ${ }^{11}$. Mais il faut également manier cette idée avec précaution dans l'étude de la grammaire technique, même après ces adaptations tardives qui favorisent l'idée d'un langage commun. La grammaire technique peut bien puiser dans les ressources de la logique pour y trouver une classification des "choses", des signifiés, ou encore les ferments d'une sémantique, mais il lui appartient aussi de développer une approche

In Categorias, CAG IV/1, p. 96, 20-21 ( l'homme "indique la qualité substantielle et la communauté des substances sous-jacentes”) ou encore à la formule de l'Isagogè: "En effet même si l'on assume 'homme' comme une qualité (poion), il ne saurait être simplement qualité, mais seulement en tant que les différences en s'ajoutant à un genre l'ont fait exister", CAG IV/1, p. 18, 18-19, trad. A. de Libera et J. -Ph. Segonds, Paris, 1998, p. 22-23.

11 "Lappellation ne signifie donc pas seulement des qualités. Il est évident que la définition diogénienne de l'appellation comme signifiant la qualité commune est indigente, et qu'elle tomberait plus sûrement que les catégories d'Aristote sous le coup de l'accusation d'incomplétude. Il se peut que les définitions qui nous soient parvenues soient incomplètes, ce qui pourrait très bien s'expliquer si Diogène de Babylonie avait seulement voulu distinguer les noms des appellations en disant que les uns signifiaient des qualités propres et les autres des qualités communes, sans limiter la signification des appellations aux qualités. Les appellations peuvent signifier les trois catégories autres que le sujet [...] Les catégories apparaissent donc bien comme relevant de la physique. Il s'agit probablement d'une typologie des différents affects d'un substrat corporel. Il est donc probable que ce système apparut d'abord dans la physique stoïcienne. Mais si l'on veut définir sémantiquement les noms communs, il faut faire appel à ce système, ce dont la citation de Diogène de Babylonie définissant l'appellation par la signification de la qualité n'est probablement qu'un écho affaibli et partiel" (Gourinat, 2000: p. 135). 
proprement métalinguistique de certaines notions. C'est le cas de l'analyse de la signification de la substance: il peut certes s'agir du fait que ce qui est désigné est une substance, et qu'elle est ou non désignée en tant que substance, mais la substance désigne aussi d'une propriété de signification ${ }^{12}$. Il s'agit alors du fait que ce qui est signifié fait l'objet d'une ostention (deixis pronominale) qui se réfère à l'existence en chair et en os de la chose, une théorie issue de l'héritage stoïcien qu'on observe de manière privilégiée dans la division des propositions définies (construites avec une articulation définie, i.e. un "pronom"), intermédiaires (construites avec un nom, i.e. un "nom propre", ou une appellation) ou indéfinies (construites avec une particule indéfinie comme tis) ${ }^{13}$. Seules les propositions construites avec un déictique sont "définies", non pas parce qu'elles identifient avec certitude un référent unique (ce que peut accomplir le nom (propre) une fois écartée tout phénomène d'homonymie) mais parce qu'elles garantissent, par leur sémanticité même, la présence en chair en os de l'objet de la deixis pronominale ${ }^{14}$. La "signification de la substance" désigne chez Apollonius une propriété de signification attachée à une partie du discours, le

12 Les grammairiens médiévaux ajouteront une autre manière de comprendre la signification de la substance comme propriété de signification, propre cette fois-ci aux noms: il s'agit de signifier une chose, quelle qu'elle soit, substance ou accident, en tant que substance; elle est souvent utilisée, notamment par Pierre Hélie, dans le but de restaurer l'unité grammaticale du nom contre une interprétation "catégorielle", trop restrictive, de la substance (Brumberg-Chaumont, 2009; 2011).

13 Voir Sextus Empiricus, Adv. Math, VIII 96-98 (traduction anglaise et texte grec R. G. Bury, Londres, 1967, pp. 287-288; traduction française J. Brunschwig et P. Pellegrin [Long; Sedley, 2001: p. 105-106]).

14 Comme le montre le témoignage d'Alexandre d'Aphrodise à propos de l'analyse du conditionnel: "Si Dion est mort, celui-ci est mort" - voir sur ce thème Brunschwig (1984; 1995: p. 115-139) et Gourinat (2000: p. 120), avec la bibliographie afférente. Nous ne suivons pas sur ce point R. Goulet lorsqu'il affirme que la proposition tire son caractère défini de sa capacité d'identification: "La proposition n'est donc définie que si elle permet d'identifier — avec plus ou moins de précision — son sujet.” (Goulet, 1978). Dans la même perspective, nous ne pensons pas que les notions de proposition définie et intermédiaire aient fait l'objet d'une confusion dans l'Antiquité, en particulier chez Ammonius, comme le suggère R. Goulet. Au contraire, la remarque de Théophraste sur la proposition singulière aristotélicienne comme proposition définie indique une conception différente de la notion de définition, conforme à la logique aristotélicienne, où la définition comme "identification" est fondamentale aux dépens de la notion de définition comme "ostension". Seule une minoration du sens de "définition" comme "identification" peut expliquer le fait que les deux propositions construites avec les expressions capables d'identifier leur sujet, les déictiques et les noms, ne soient pas équivalentes logiquement, et qu'ainsi la ligne de démarcation fondamentale de la 
pronom, une propriété métalinguistique (la deixis) et non une propriété physique ou ontologique du signifié - être une substance, être un substrat - un trait que celui-ci possède de la même façon, qu'il soit désigné par un nom ou par un pronom. Il y a évidemment un lien entre ces différentes aspects: le pronom peut signifier pour Apollonius la même substance que signifient les noms propres sans signifier leur qualité, c'est-à-dire qu'ils peuvent signifier le substrat indépendamment de ses propriétés, parce qu'il désigne directement cette substance par ostension, ce que ne peut pas faire le nom.

La post-datation de la Technè attribuée à Denys le Thrace induit ainsi une évolution historiographique. Elle s'accompagne d'une lecture critique de certaines sources qui avaient servi pour reconstituer la théorie des catégories et la "grammaire" stoïciennes. Ce contexte éclaire d'un nouveau jour la manière dont des notions appartenant à l'ontologie aristotélicienne ou stoïcienne, telle que celle de substance, peuvent acquérir un sens technique et proprement métalinguistique. Celui-ci s'agrège certes à ce sens ontologique ou catégoriel, mais s'en distingue aussi fortement, comme un mode de signifier se distingue d'un signifié. Encore faut-il préciser que cette distinction ne sépare pas radicalement la philosophie de la grammaire, puisqu'une préoccupation proprement linguistique s'ancre dans la partie de la dialectique stoïcienne (étude des signifiants) qu'il est convenu d'appeler "grammaire stoïcienne", qu'une approche "sémantique" des problèmes philosophiques se marque de plus en plus dans l'Antiquité tardive grecque ou latine, et qu'enfin les grammairiens manient tout aussi bien des questionnements philosophiques (Blank, 1982: p. 5; Ildefonse, 1997: p. 15-16, 32-40); elle tente plutôt de capter la différenciation entre une préoccupation sémantico-ontologique et une préoccupation linguistique dotée d'un métalangage propre, au moment même où celle-ci émerge pour se fixer dans une classification des savoirs. Comment penser ce terrain commun? En termes de "transferts", d' "emprunts", de "lingua franca" ?

Suivant la proposition de F. Ildefonse ${ }^{15}$, il nous semble pertinent de s'efforcer de penser la présence d'une épistémè commune là où l'on est toujours tenté d'observer, a

classification se trace entre la proposition définie et toutes les autres, que R. Goulet souligne dès le début de son analyse.

15 Ildefonse, 1997: p. 15-16, 32-40. Citons un passage particulièrement clair sur la méthode employée: "Tout en cherchant à identifier le statut que ces pans de logique stoïcienne occupent dans le discours du grammairien, j'ai cherché à relever [...] comment ce mélange de référence exacte, de conceptualité désaffectée et d'épistémè apophantique déterminante anime les démonstrations grammaticales d'Apollonius [...]. 
posteriori, de l'éclectisme philosophique ou des transferts de "disciplines". L'Antiquité tardive est en effet marquée par des circulations terminologiques, constantes d'une secte philosophique à une autre, et d'une "discipline" à une autre. Cela ne veut pas nécessairement dire que le sens philosophique de ces notions s'exténue au fil de leurs pérégrinations. À propos des emprunts terminologiques que Porphyre fait aux Stoïciens, R. Chiaradonna revient sur la méthode de J. Barnes, qui, constatant que ces emprunts ne respectent pas la doctrine stoïcienne, en déduit que Porphyre ignore cette dernière: non sequitur, rétorque R. Chiaradonna (2008: p. 16). J. Barnes propose de considérer que l'Isagogè de Porphyre est simplement "écrit dans la lingua franca philosophique de l'époque" (Barnes, 2003: p. xix). Une approche analogue à celle de J. Barnes a été récemment adoptée par $\mathrm{S}$. Ebbesen à propos de l'utilisation de notions philosophiques chez les grammairiens, en particulier chez Priscien: ce dernier en ferait une utilisation massive, tout en ignorant l'essentiel de leur sens philosophique ${ }^{16}$

J'ai [...] essayé de montrer, suivant la méthodologie de l'archéologie du savoir, combien la syntaxe d'Apollonius participe d'une épistémè, ou d'une configuration des savoirs, qui la relie à la logique stoïcienne et au projet général apophantique, caractéristique de la philosophie grecque inaugurée par Platon, que le stoïcisme a porté à son apogée. De même que la grammaire demeurait bloquée, séparée de son émergence comme discipline autonome par une orientation normative de la philosophie, de même certaines analyses grammaticales restent déterminées souterrainement, d'autres bloquées, par des lignes de forces logiques qui circulent et se poursuivent en elles" (p. 37). Pour l'application de cette méthode à l'analyse de la grammaire d'Apollonius voir "Introduction" (p. 253 sqq.), notamment p. 255: "Détacher ainsi, sans l'abandonner, la justification de la normativité, définir la tâche grammaticale comme justification de la langue, corpus bien formé, et justifier du même geste l'originalité grammaticale qui engage une telle activité, reprendre dans les analyses grammaticales le soucis apophantique du monde sensible, c'est encore rester logicien" (nous soulignons). Voir également p. 262, p. 271, et la conclusion de l'ouvrage.

16 S. Ebbesen considère que les notions philosophiques présentes chez Priscien, en particulier celles issues des Catégories, sont très peu comprises, et utilisées avec un piètre discernement par le grammairien latin (Ebbesen, 2009). Il nous semble que ce que M. Baratin à montré à propos des deux sens de "substance" et de "qualité" dans les Institutions, ou l'étude fine d'A. Garcea sur substance et accident (voir infra n. 19) constituent des éléments suffisamment importants et cohérents avec l'héritage philosophique des commentateurs de l'Antiquité tardive pour motiver non seulement une autre approche des sources philosophiques de Priscien, qui soit elle-même philosophique, mais aussi une réflexion sur le caractère philosophique de l'entreprise grammaticale, par-delà la seule question des "sources". 
La position de J. Lallot semble hésiter entre indifférence ${ }^{17}$, "dépendances" et "distorsions", à vrai dire davantage lexicales que notionnelles ${ }^{18}$.

R. Chiaradonna conteste la pertinence de la notion de lingua franca pour saisir les rapports de Porphyre au Stoïcisme ancien, dans un argumentaire qui prend notamment l'usage du terme hupostasis dans des contextes philosophiques différents, y compris en grammaire:

Indeed, countless instances are known in which Stoic terms are adopted without any trace of their original philosophical meaning; but in many cases Stoic theories are neither faithfully reported nor ignored. Rather, such doctrines are integrated in a different philosophical framework: they are adapted and 'misunderstood'; in turn, Stoic thought alters those doctrines by which it is assimilated. [Barnes].'s scheme fails to grasp the subtleties of such a complex process of interaction. The very notion of a "philosophical lingua franca" is highly controversial. The fact, for instance, that the same term or argument occurs in Porphyry and in Galen, Sextus, Alexander and Grammatical texts does not in itself prove that was not employed by Porphyry in a distinctive philosophical manner. Some interpreters have sought to infer philosophical influences from terminology. While [Barnes] is correct in rejecting a similar approach, his alternative hypothesis (that Porphyry adopted a neutral lingua franca in which terms and arguments - whatever their origin may have been - had lost their distinctive philosophical connotation) is equally unconvincing. It is certainly wrong to posit Stoic influences on the mere basis of terms like hupostasis. Yet the situation may vary: the mere fact that a commonly shared philosophical terminology existed does not imply that different authors (or the same author in different contexts) always made a use of a neutral lingua franca. Rather, one should seek to detect if (and where) current terms and arguments are

17 "En présence de cette diversité [le nom signifiant parfois la substance avec la qualité parfois la qualité chez Apollonius], on fera remarquer à juste titre que ousía renvoie plutôt à l'aristotélisme et poiotès, avec ses deux variétés 'propre' et 'commune', plutôt au Stoïcisme, mais est-ce bien important pour la théorie grammaticale?" (Lallot, 1998: p. 38).

18 "Il reste cependant que certains domaines de la grammaire gardent, au moins au niveau du vocabulaire, sinon des concepts sous-jacents, la marque d'une théorie philosophique. Dans ces cas, bien sûr, je me suis efforcé de montrer les dépendances, et éventuellement aussi les distorsions" (Lallot, 1998: p. 39; voir Sluiter, 1990: p. 40-41). 
used in a philosophically distinctive way (and ascertain just what that way might be) (Chiaradonna, 2008: p. 16).

On pourrait en dire autant de ousia, sumbébèkos, poiotès, hupokeimenon, koinôs poion, idiôs poion, etc., et tenter d'appliquer, mutatis mutandis, le cadre méthodologique proposé par R. Chiaradonna à la question des relations entre grammaire et philosophie. L'emploi d'un terme philosophique n'est pas certes en soi la garantie d'une influence philosophique, et, d'une manière générale la question des rapports entre philosophie et grammaire ne saurait se limiter à un repérage des "sources philosophiques" chez tel ou tel grammairien. Il convient de faire l'hypothèse d'une adaptation, d'une "mécompréhension" elle-même philosophique, propre à chaque auteur sur la base d'une terminologie commune, une phénomène largement répandu dans l'Antiquité tardive, et commun à toutes sortes de textes, qu'ils soient logiques, grammaticaux, ou rhétoriques. Cela implique de prendre au sérieux les éléments philosophiques que nous trouvons chez nos grammairiens, non seulement parce que la partition des "disciplines" demeure factice, même après l'émergence d'une grammaire technique, quelles que puissent être ses déclarations de principes sur son indépendance, mais aussi parce que le discours grammatical ne fait pas que manipuler aveuglément des notions philosophiques vidées de leur contenu; il peut être une source importante pour la connaissance des doctrines anciennes, le lieu d'une élaboration philosophique propre, faite d'emprunts et de déplacements, comme c'est le cas de tout texte théorique ${ }^{19}$.

19 La question de l'énoncé, comme celle du nom et de sa signification, en est un exemple caractéristique, voir l'article récent d'A. Garcea sur les notions de substance et d'accidents chez Boèce et Priscien (Garcea, 2009), ou encore les travaux de M. Baratin sur la structure substance/qualité qui se substitue dans la grammaire latine, pour tous les niveaux d'analyse de l'énoncé (y compris le nom lui-même), à la structure aristotélicienne sujet-prédicat (Baratin, 1989: p. 377 sqq.; 1994: p. 49-79). F. Ildefonse développe une analyse très différente de la question de l'énoncé en grammaire et en logique par sa méthode et son objet (la grammaire d'Apollonius et ses rapports au Stö̈cisme ancien), en observant de manière privilégiée une "epistémè apophantique" commune, qui détermine de manière radicale les conditions d'émergence de la grammaire grecque technique (voir supra n. 15). Quant à J. Lallot, dans une perspective comparable, il n'hésite pas à reconnaître derrière le "noyau phrastique" constitué par un nom et un verbe le "syntagme sujet-prédicat, héritier en ligne directe" de l'énoncé minimal chez Platon, Aristote ou encore les Stoïciens anciens (Lallot, 1997: I, p. 33, n. 58), justifiant ainsi l'emploi d'un méta-langage logico-philosophique qui n'est pas celui d'Apollonius. 


\section{La reconstitution de la théorie apollonienne du nom et du pronom: plusieurs sens de "substance" ?}

À ces difficultés méthodologiques s'ajoute l'obstacle d'un corpus lacunaire. La partie de la grammaire d'Apollonius portant sur les parties du discours nous est partiellement parvenue. Manque en particulier la partie sur le nom, qui nous aurait délivré la formulation apollonienne du propre du nom comme partie du discours - sa propriété de signification - ainsi que sa définition. Il nous reste à reconstituer cette doctrine sur la base de ce que nous trouvons, de manière assez éparse, dans la Syntaxe et dans le Peri antônumias, ainsi que dans les reconstructions que l'érudition des siècles passés nous ont offertes. Or ces différentes sources ne convergent pas parfaitement.

Plusieurs niveaux d'analyses sont à prendre en compte. À un premier niveau de lecture, fondé sur l'enseignement général de la Syntaxe, on peut dire que la signification de la substance est réservée aux seuls pronoms, tandis que la signification de la qualité concerne les noms. Cette "répartition" des tâches à propos d'un même sujet (hupokeimenon) assure la complémentarité des deux parties du discours, y compris à la troisième personne - où noms et pronoms sont possibles. On observe ainsi un lien étroit entre signification de la substance et deixis, à laquelle les noms n'ont pas part (voir infra §3).

Mais les choses se compliquent, même dans le cadre de la seule lecture de la Syntaxe, comme on le voit au début du traité, lorsqu'il s'agit de traiter des inquisitifs. À propos de la distinction de la série des inquisitifs nominaux et adverbiaux, Apollonius s'interroge sur la raison d'être de plusieurs inquisitifs parmi les inquisitifs nominaux, l'inquisitif "de substance" (qui?), et les autres inquisitifs, de "qualité" (quel?), de "quantité" (en quelle quantité?), etc:

$\S 30$... Pourquoi sont-ils non pas un seul du côté du nom et un seul du côté de l'adverbe, mais plusieurs - d'un côté: tís [qui?], poîos [quel? (qualité)], pósos [en quelle quantité?] ... Quant à l'existence de plusieurs nominaux et de plusieurs adverbiaux, en voici la raison. $\S 31 \ldots$ Quand on s'enquiert de l'identité (huparxis) d'un sujet (hupokeimenon), on emploie tís [qui?] [...] Les réponses sont alors nominales, formées d'un appellatif ou d'un nom propre (les noms propres exprimant également la substance <générique $>$ ); on peut répondre en effet: "un homme / un cheval / Tryphon (ce dernier incluant l'homme) marche". On peut aussi employer le mot qui remplace le nom (j'entends le nom propre), ainsi quand on répond 
"moi". § 32 Mais comme les noms donnés en réponse ne dévoilent pas les accidents - aussi bien le nom tís n'interroge-t-il que sur la substance, à quoi s'ajoutent la qualité, la quantité, la taille - on en a inventé en plus de quoi questionner sur ces accidents ${ }^{20}$.

Seuls certains noms, ceux qui signifient la substance, et les pronoms peuvent répondre à l'inquisitif de substance. Même si Apollonius ne le dit pas ici explicitement, il semble bien qu'il y ait une classe de noms qui signifient la substance, comme "homme", "animal", et les noms propres, comme "Socrate"; les noms qui répondent aux inquisitifs portant sur la qualité ou la quantité sont ce qu'on appellera plus tard des "adjectifs", comme "blanc", "grand". Si tous les noms signifient la qualité d'un sujet et si certains noms signifient la substance de ce même sujet, il faut bien admettre que certaines "qualités" sont en réalité des "substances", ce qui nous ramène aux deux sens de "qualités" distingués par $\mathrm{M}$. Baratin à propos de Priscien. C'est bien ainsi que ce passage de la Syntaxe sur la série des inquisitifs a été compris par M. Baratin:

La double acception des termes ousia et substantia est assurément présente chez Apollonios, mais de façon beaucoup moins développée que chez Priscien. Chez Apollonios en effet l'ousia apparaît essentiellement porteuse de la 'signification substantielle' [i. e. la substance-substrat, la substance au sens 1], c'est-à-dire la signification qui est exprimée de façon globale à la fois par le nom et par le pronom ${ }^{21}$. La 'signification substantive' de l'ousia [i. e. celle où l'ousia désigne une certaine qualité, la substance au sens 2], celle qui est portée seulement par certains noms, n'apparait que dans un contexte précis, celui des interrogatifs. Chez Priscien en revanche ces deux acceptions sont constamment concurrentes [...] Apollonios ne

20 GGII/2, p. 28, 3-30, 2; Lallot, 1997: I, p. 104-105. D’une manière générale, toutes les traductions citées ici ont été légèrement adaptées dans le sens de la littéralité. Une partie de cette citation est reprise infra, Texte 2 .

21 L'ensemble de l'interprétation que nous proposons ici, et que nous avons déjà précisée à propos de Priscien dans notre étude précédente (Brumberg-Chaumont, 2009) implique de revenir sur l'amalgame entre la signification de la substance-substrat par tous les noms et la signification de la substance comme propriété de signification chez les pronoms. Nous y revenons tout au long du présent exposé. 
représente que l'amorce du développement des notions de substantia et de qualitas qu'on observe chez Priscien ${ }^{22}$.

Or nous savons que les inquisitifs comme "tís (qui?)" sont pour Apollonius des noms et non des pronoms, un point sur lequel Apollonius est très clair; c'est même tout le sens du texte du Peri antônumias qui mentionne la même série des inquisitifs, sur lequel nous revenons plus loin en détail. Ce n'est pas parce qu'il signifie la seule substance que tís est un pronom (voir infra Texte 13).

Les noms qui signifient la seule substance ne peuvent par conséquent pas la signifier dans le même sens où les pronoms signifient la substance à titre de propriété de signification: les uns signifient la seule substance à titre de signifié à l'exclusion de tout accident du sujet, tandis que les autres signifient la seule substance à titre de propriété de signification, à l'exclusion de toute qualification du sujet, qu'elle soit substantielle ou accidentelle (même si les propriétés du sujets puissent être visées conjointement dans la deixis pronominale; voir infra § 4.3). Il apparaît donc qu'il convient de distinguer pour Apollonius entre la signification de la substance comme propriété de signification, qui s'attache en propre au pronom, et la signification de la substance comme caractéristique du signifié, qui s'attache à certains noms, les noms de substances, un sens de "substance" qui nous ramène à une certaine "qualité", une qualité substantielle. Les noms qui signifient la substance le font non pas parce qu'ils possède le mode de signifier du pronom, l'équivalent de la possession de la deixis, mais parce qu'ils signifient la substance du sujet, et non pas un accident.

Mais si le sens de "substance" porté par le texte d'Apollonius sur les inquisitifs de substance dans la Syntaxe semble bien correspondre à la substance au sens 2 dégagée par M. Baratin, la substance comme "qualité substantielle", il n'est pas explicitement distingué du sens de "substance" au sens 1, le substrat des qualités, représenté dans notre texte par le "sujet" (hupokeimenon). Il est en revanche nettement distingué de la substance comme propriété de signification des pronoms dans le Peri antônumias : c'est bien le sens de la polémique du grammairien alexandrin avec ceux qui veulent identifier "tís" à un pronom au prétexte qu'il signifie exclusivement la substance (voir infra § 5). "Tís" signifie en effet la seule substance, par opposition aux accidents signifiés par les autres inquisitifs nominaux, et non la

22 Baratin, 1989: p. 405. À vrai dire M. Baratin parle de deux sens de "substance" et non de deux sens de "qualité", mais il veut simplement dire qu'il y a un sens de "substance" qui veut dire "qualité substantielle" chez Apollonius. 
substance liée à la deixis pronominale et à la détermination des personnes, propre aux pronoms comme partie du discours.

Si M. Baratin a donc raison de souligner qu'il existe également plusieurs sens de "substance" chez Apollonius, il faut insister sur le fait que les deux sens de "substance" mobilisés par les textes sur les inquisitifs de substance dans la Syntaxe et le Peri antônumias ne sont pas équivalents aux deux sens de "substance" suggérés par M. Baratin, car ceux-ci étaient internes à la classe nominale. Ils visent ici à différencier d'une part deux parties du discours, les noms et les pronoms, et d'autre part, les noms qui signifient la substance des autres noms, qui signifient les accidents.

Pourquoi faudrait-il alors distinguer chez Apollonius un autre sens de "substance", correspondant à la substance-substrat des qualités (substance au sens 1)?

C'est la question posée par la présence dans le Peri antônumias de l'idée que les noms signifient "la substance avec la qualité", tandis que les pronoms signifient seulement la substance. Cette formule est reprise dans de nombreuses études sur la grammaire d'Apollonius comme une variante ou un complément par rapport à l'héritage stoïcien, tel qu'on l'observe dans la Syntaxe, lequel attribue aux noms la signification de la qualité (voir infra § 4.4). Comment comprendre le rapport entre ces deux textes?

Il faut reconnaitre d'abord que même dans le cadre de la théorie "standard" de la Syntaxe, où les noms signifient la qualité et les pronoms signifient la substance, il est tout aussi peu probable qu'il l'était chez les Stoïciens anciens de dire que pour Apollonius les noms ne signifient que les propriétés des choses, et non les choses elles-mêmes, les "porteurs" (hupokeimena) des qualités. Ils servent certainement à les désigner et ce sont eux qu'ils nomment, pas leurs propriétés. Bien que le substrat des qualités ne soit pas appelé "substance" dans les textes centraux de la Syntaxe, il est assez plausible de voir dans l'hupokeimenon d'Apollonius l'équivalent de la substance chez Priscien. De fait la plupart des commentateurs anciens et modernes ont vu chez Priscien une équivalence entre la "substance" (substantia) du propre du nom ("signifier la substance et la qualité") et les "sujets" (subjecta = hupokeimena) auxquels les qualités communes ou propres sont distribuées dans la définition du nom - définition attribuée à Apollonius lui-même, suivant les Scholies à la Technè ( voir infra Texte 11) L'existence d'une notion de substance-substrat, exprimée par le terme hupokeimenon dans l'analyse de la signification des noms, semble assez indiscutable. Mais la formule du Peri antônumias nous conduit en outre à considérer la possibilité que cette notion soit aussi exprimée par Apollonius avec le vocabulaire de l'ousia, et non pas seulement de l'hupokeimenon. À ces éléments tirés des passages 
centraux de la Syntaxe et de la formule contenue dans Peri antônumias, s'ajoute la nécessité de prendre en compte la présence dans la Syntaxe d'autres textes, délicats à interpréter, mais qui semblent aller dans le même sens, c'est-à-dire l'attribution à Apollonius d'une signification de la substance et de la qualité à tous les noms (voir infra $\$ 4.1$ et 4.2).

Ces différentes données, aussi problématiques qu'elles soient, justifient une interrogation sur l'existence chez Apollonius chez Apollonius Dyscole de trois notions distinctes de substance - sinon trois sens d'ousia, puisqu'il s'avère que le terme hupokeimenon est le plus souvent réservé à la substance-substrat, sans que le vocabulaire de l'ousia ne soit toutefois complètement absent.

Nous rappelons d'abord les quelques textes qu'il est possible de glaner dans la Syntaxe à propos de la signification des noms et des pronoms, afin de dresser un premier tableau de la doctrine générale d'Apollonius, puis nous revenons sur certains passages délicats de la Syntaxe qui semblent attribuer aux noms la signification de la substance, outre celle de la qualité, pour enfin revenir en détail sur le contexte argumentatif dans lequel est avancée l'idée que les noms signifient "la substance avec la qualité" dans le Peri antônumias.

\section{Les noms signifient la qualité du sujet (bupokeimenon), les pronoms signifient la substance du sujet}

Commençons par quelques brefs rappels sur la doctrine d'Apollonius fondés sur quelques passages clefs de la Syntaxe et d'autres textes du grammairien grec:

Texte 1 (Syntaxe II, § 22)

Linstitution des noms a été inventée pour [signifier] des qualités, communes, e.g. homme, ou particulières, e.g. Platon ${ }^{23}$.

Texte 2 (Syntaxe I, §31)

Quand on s'enquiert de l'identité (huparxis) d'un sujet (hupokeimenon), on emploie tís [qui?] ... Les réponses sont alors nominales, formées d'un appellatif ou d'un nom propre (les noms propres exprimant également la substance < générique>; on peut répondre en effet: "un homme / un cheval / Tryphon (ce dernier incluant l'homme) marche". On peut aussi employer

23 GG II/2, p. 142, 1-2; Lallot, 1997: I, p. 152. 
le mot qui remplace le nom (j'entends le nom propre), ainsi quand on répond "moi" 24 .

\section{Texte 3 (Syntaxe I, §78)}

Le nom conserve à tous les cas ce qui fait sa propriété: la variation personnelle ne fait pas partie de sa propriété, même s'il peut être employé à la première ou à la troisième personnes; ainsi dans eimì Odusseús [je-suis Ulysse] et à la deuxième personne, Odusseùs ê̂ [tu-es Ulysse], "Ulysse" ne cesse jamais [de signifier] la qualité particulière ${ }^{25}$.

\section{Texte 4 (Syntaxe I, § 112)}

C'est que les noms propres, qui portent leur particularité (idiotèta) en euxmêmes, n'ont pas le même besoin de l'article que ceux qui dénotent une notion commune (koinèn einoian). En effet, c'est comme une contraction du large [champ] de la qualité qu'opère l'apposition de l'article ${ }^{26}$.

\section{Texte 5 (Syntaxe I, §120)}

Il ressort clairement de là que, quand nous employons un nom [dans la question] ${ }^{27}$ nous nous enquérons de la substance (ousia) du sujet (hupokeimenon) : c'est elle et elle seule que désignent les pronoms (même si la deixis qu'ils opèrent oriente également vers les accidents (sumbébèkonta), d'où il suit qu'ils s'appliquent à n'importe quel sujet. Quand

24 GG II/2, p. 29, 1-8; Lallot, 1997: I, p. 105.

25 GG II/2, p. 66, 8-12; Lallot, 1997: I, p. 121.

26 GG II/2, p. 95, 3-5; Lallot, 1997 : I, p. 132.

27 Le paragraphe précédent (Syntaxe I, § 119) montre qu'Apollonius s’intéresse ici précisément aux questions où l'interrogatif "qui?" est associé ou bien à un nom propre par l'intermédiaire d'un verbe "d'identité", "substantielle" ou "onomastique", comme "être" ou "s'appeler", ou bien à un pronom - en ce cas, même si Apollonius ne le précise pas dans ce passage, seul un verbe d'identité "substantielle" est possible (voir infra $§ 4.2$ ). Dans le texte suivant, Apollonius est passé aux cas où "qui?" s'emploie seul et peut alors être associé à n’importe quel verbe, comme il le précise au début du $§ 121$. Dans les deux cas, lorsqu'il est employé seul ou lorsqu'il est associé à un nom ou à un pronom dans la question, la question porte que l'identité (huparxis) du sujet, sa substance, et non ses accidents, comme nous le montre les textes d'Apollonius sur les série des inquisitifs nominaux. Pour la traduction de huparxis par "identité" dans les deux types d'occurrence, voir infra note 61. 
au contraire nous employons un pronom, c'est que nous avons déjà prise sur la substance, mais plus du tout sur la propriété (poiotès) qui s'y ajoute suivant l'institution du nom ${ }^{28}$.

\section{Texte 6 (Syntaxe I, §121)}

Si on répond à ces questions [i. e. avec tis seul] au moyen d'un pronom, il n'y a plus besoin ensuite d'une autre question - par exemple, si on dit "moi", ou "lui", car ces pronoms désignent des personnes déterminées. En revanche, avec les noms, du fait qu'ils sont exposés à l'homonymie, la réponse n'est pas déterminée au même degré: si en effet on répond "Ajax", à cause de l'homonymie dont nous avons parlé, on se verra répliquer: "lequel des deux?". Et à cette dernière question on répondra [en énonçant] la propriété (idiotèta) qui est attachée à l'un d'eux: «le grand» ou «le petit fils de Télamon» ${ }^{29}$.

\section{Texte 7 (Syntaxe II, § 41)}

De fait, potentiellement, c'est un nom propre qu'on donne à entendre quand on emploie un pronom, pas le nom sous sa forme phonétique, mais ce qui est l'objet de la deixis pronominale, à savoir la qualité propre du sujet ${ }^{30}$.

\section{Texte 8 (Syntaxe II, §45)}

Les pronoms [de la troisième personne] doivent donc leur naissance non au fait que les noms ne seraient pas recevables à cette personne, mais au fait qu'ils n'ont pas de part à la deixis, qui justement réside dans les pronoms ${ }^{31}$.

Texte 9 (Syntaxe III, § 96)

[...] dans Aías, l' "un" est, comme signifié conjoint (paruphistamenon), associé à la qualité particulière (idiôs poion)» ${ }^{32}$.

Texte 10 (Peri antônumias)

28 GG II/2, p. 101, 11-102, 3; Lallot, 1997 : I, p. 135.

29 GG II/2, p. 102, 8-14; Lallot, 1997 : I, p. 135.

30 GG II/2, p. 155, 3-5; Lallot, 1997 : I, p. 158.

31 , GG II/2, p. 159, 1-3; Lallot, 1997 : I, p. 159.

32 GG II/2, p. 352, 5-6; Lallot, 1997 : I, p. 238. 
Les pronoms ne remplacent ni les noms appellatifs, ni les épithètes, dans la mesure où ils déterminent quelque chose d'un (hen ti), alors que le nom appellatif s'applique à une pluralitée ${ }^{33}$.

\section{Texte 11 (Fragmenta: Peri onomatôn)}

Apollonius et Hérodien définissaient ainsi le nom: "une partie de phrase casuelle assignant une qualité, commune ou particulière, à chacun des sujets (hupokeimenôn), corps ou choses. Mais Romanos et Philopon remplacent "qualité" par "substance" ${ }^{34}$.

Apollonius hérite de la "grammaire stoïcienne" la notion de qualité particulière, désormais bien ancrée dans son opposition avec la qualité commune (voir Texte 1). Conformément à ce que nous indique le témoignage de Diogène de Babylonie (reflétant probablement un état tardif de la doctrine stoïcienne), le nom signifie la qualité, commune ou propre. Le nom propre ne correspond plus à un type de signifiant particulier, puisqu'il y a sous-catégorisation de l'appellation sous le nom, qui donne lieu à la distinction entre nom propre et nom appellatif. L' "articulation définie" des Stoïciens devient le pronom, à quoi l'on attribue une capacité déictique, une désignation de la pure substance; le pronom se substitue au seul nom propre (Texte 11). Il existe une variante à cette formulation stricte: le pronom peut se substituer à l'appellatif si celui-ci est déterminé par un article, et seulement dans la mesure où est compris à sa place un nom propre (Texte 4).

La capacité du nom à identifier un individu précis lui vient du lien unique et exclusif entre celui-ci et la qualité particulière. Cette capacité lui est commune avec le pronom, qui opère la même identification du fait de la deixis qu'il comporte. Lidentification est bien plus fiable dans le cas des pronoms car il faut compter avec l'homonymie des noms propres, mais cela ne change rien au principe d'une même identification unique, sur la base de deux comportements sémantiques distincts. Sur le principe, en effet, il n'y a qu'une seule qualité particulière par individu, même si, accidentellement le même nom a été donné à deux individus. On peut toujours dissiper l'homonymie par l'adjonction d'une caractéristique supplémentaire, comme "le grand", "le fils de Télamon" (Texte 6). Ce point commun entre noms propres et des pronoms montre que la compréhension de la

33 GG II/1, p. 26, 9-11; Ildefonse, 1997 : I, p. 307.

34 GG II/3, p. 38 (= GG I/3, p. 524, 7-11). 
notion de "détermination/définition" comme "identification" a pris le pas chez les grammairiens sur la détermination comme "démonstration" (deixis), telle qu'on l'observe dans le Stoïcisme ancien. Comme on l'a vu, seuls les pronoms permettaient en effet de construire des propositions définies, du fait de leur fonction déictique, les noms et les appellations formant des propositions seulement "intermédiaires", sans que la relation unique et exclusive entre le porteur et son nom ne confère à ce dernier de privilège logique par rapport à l'appellation. Chez Apollonius, comme dans le Stoïcisme ancien, les noms, fussent-ils propres, n'ont pas part à la deixis, et de ce fait, n'identifient pas une "personne" (i. e. un acteur de la situation d'interlocution). Le nom propre est une sorte d'intermédiaire entre l'appellatif (avec lequel il partage la signification de la qualité), et le pronom (avec lequel il partage la capacité à identifier un individu et un seul). L’unité est attachée à la qualité particulière signifiée par le nom propre comme une "consignification" (Ildefonse, 1997: p. 264 sqq.), tandis qu'une notion commune et la pluralité est attachée à la qualité commune signifiée par l'appellatif (Textes 4, 9 et 11).

La Syntaxe montre qu'il y a complémentarité des noms et des pronoms, non seulement à la première et à la deuxième personne, dans la mesure où les noms ne pouvaient occuper ces places, mais également à la troisième personne, à laquelle les noms prennent part. Les pronoms à la troisième ne sont pas pour autant superflus car ils possèdent une puissance déictique que n'ont pas les noms qu'ils remplacent, c'est-à-dire les noms propres (Texte 8). C'est ainsi que pronoms, noms propres et certains noms appellatifs donnent des informations complémentaires en réponse à l'interrogatif de substance "tís (qui?)" : les pronoms indiquent la substance du "sujet", hupokeimenon) tandis que les noms signifient la qualité propre ou la qualité commune - du moment qu'elle est, comme nous l'avons vu, "substantielle", ce qu'Apollonius ne dit cependant pas explicitement. Quand les interrogatives contiennent un nom, elles portent sur la substance, la qualité étant déjà connue, tandis que lorsqu'elles contiennent un pronom elles portent sur la qualité du sujet, la substance étant déjà connue (Textes 1 et 5).

\section{Signification de la qualité, signification de la substance, signification de la substance avec la qualité}

Plusieurs éléments à l'intérieur de la Syntaxe viennent compliquer l'opposition binaire entre la signification de la substance et celle de la qualité $e^{35}$. Ainsi, le texte

35 F. Ildefonse parle à ce propos d' "abstraction" (Ildefonse, 1997: p. 305), qu'elle oppose au point de vue du procédé concret d'identification d'un référent: nous y revenons plus loin. 
7, semble indiquer que l'objet de la deixis pronominale n'est pas la pure substance, mais la qualité particulière du référent. De même, le texte 5 suggère que le pronom désigne des "accidents", outre la simple substance. Inversement, la Syntaxe affirme que l'existence substantielle est impliquée dans le fait d'être nommé, mais non l'inverse: quand on a "ceci", on n'a pas nécessairement "Ulysse", mais quand on a "Ulysse", on a nécessairement "ceci", ce qui semble indiquer que le nom signifie la substance et la qualité, et le pronom la seule substance (voir infra Texte 13). Dans un esprit comparable, le même texte 5 semble suggérer que la substance du sujet est présupposée par l'institution du nom, puisqu'elle lui surimpose une propriété. Quant à la définition du nom attribuée à Apollonius par les Scholies, elle mentionne bien deux corrélats sémantiques pour le nom, soit "les sujets, corps ou choses" outre les qualités assignées (Texte 11). Le texte 2 affirme que le nom signifie une substance (en l'occurrence générique), tandis que Choeroboscos (Texte 12 infra) attribue à Apollonius la signification de la "substance" commune ou particulière par les noms, en lieu et place de la "qualité" mentionnée par les Scholies (Texte 12).

Il est donc nécessaire de faire droit à ces éléments divers, et de tenter de dénouer tous les fils de ces données dissonantes, sinon divergentes.

Certains exégètes contemporains donnent un poids doctrinal à l'idée que les noms signifient la substance, au moins égal à celui accordé aux multiples indications contenues dans la Syntaxe en faveur de la signification de la qualité. La situation est en réalité assez confuse ${ }^{36}$. Deux passages de la Syntaxe semblent venir apporter un appui

36 Voir les tentatives de J. Lallot pour démêler les différents fils qu'offrent la position théorique d'Apollonius, "bancale" selon lui, sur la signification des noms, entre substance et qualité, traditions stoïcienne et aristotélicienne, noms, adjectifs et pronoms (Lallot, 1997: t. II, p. 100, n. 84). Même présentation de l'hésitation entre qualité et substance avec la qualité chez M. Baratin (2006: p. 233). À propos de la préséance du nom sur le verbe fondée sur le fait que l'institution des noms porte sur les corps (Texte 1), F. Ildefonse attribue également à Apollonius la signification de la substance et la signification de la qualité dans un argumentaire où l'articulation entre les deux aspects de la signification des noms n'est pas parfaitement explicite. Pour illustrer "l'ancrage du privilège grammatical du nom sur le verbe dans une métaphysique de la substance”, elle cite la définition du nom selon Choeroboscos, où le nom signifie la substance, qu'elle juge "apollonienne": "Une partie de phrase qui pour chacun des corps donnés ou pour chacune des actions assigne la substance commune ou propre' [...] le nom a préséance sur le verbe en tant qu'il signifie la substance, et le verbe l'accident" (Ildefonse, 1997: p. 290-291), et conclut: "Substance et accident dans la théorie physique et métaphysique péripatéticienne, corps et disposition de ces corps dans la physique stoïcienne, servent, pour distincts qu'ils soient, la même préséance du nom sur le verbe" (id., ib.: p. 291- 
particulier à l'idée que le nom signifie la substance (et la qualité) pour Apollonius, tandis que deux autres affirment en outre que le pronom signifie les accidents.

\subsection{Le nom signifie la substance (générique)}

F. Ildefonse propose de voir une équivalence entre le sujet (hupokeimenon) - le "référent" dans la traduction de J. Lallot —, la substance (ousia), le tode ti aristotélicien et la substance générale (genikè ousia) dont Apollonius dit qu'elle est exprimée par les noms propres "soit l'approche la plus générale, la plus indéterminée, la moins riche de détermination discursive, celle de l'existence d'une substance" (Ildefonse, 1997: p. 303; voir 1999, p. 163). Le fait que le nom propre exprime la substance générique serait une pièce à porter au dossier en faveur de la signification par le nom de la substance (outre la qualité). Nous rappelons le texte en question (Texte 2):

Quand on s'enquiert de l'identité (huparxis) d'un sujet (hupokeimenon), on emploie tís [qui?] ... Les réponses sont alors nominales, formées d'un appellatif ou d'un nom propre (les noms propres exprimant également la substance < générique>; on peut répondre en effet: "un homme / un cheval / Tryphon (ce dernier incluant l'homme) marche". On peut aussi employer le mot qui remplace le nom (j'entends le nom propre), ainsi quand on répond "moi"37.

L'exemple donné, qui affirme que "Tryphon" inclut l'homme, laisse penser que la substance générique ne serait rien d'autre qu'un genre, ou plus exactement ici une espèce, l'homme. C'est bien l'interprétation retenue par J. Lallot, en note de sa traduction:

Lidée, illustrée par ce qui suit immédiatement, est que le nom propre, en désignant un individu particulier, fait aussi implicitement référence

292), tout en considérant qu'Apollonius réserve, dans un esprit stoïcien, la signification de la qualité au nom: "Définir, comme il le fait, nom commun et nom propre en termes de qualité, réserver le terme d'ousia ou d'hupokeimenon au signifié du pronom, confirme encore sa parenté avec le stoïcisme” (id., ib.: p. 290). Larticulation entre ces deux aspects réside en fait dans la notion de "présupposition" (id., ib.: p. 300 sqq.) comme nous allons le voir plus loin.

37 GGII/2, p. 29, 1-8; Lallot, 1997: I, p. 105. 
à la classe à laquelle il appartient et que désigne normalement l'appellatif: "Tryphon" implique "homme" (Lallot, 1997: II, p. 27).

Reste la mention de la substance, en lieu et place de la qualité, signifié habituel des noms, mention dans laquelle J. Lallot voit des "relents aristotéliciens" (Lallot, 1997: II, p. 27). Si l'on retient l'influence prédominante de la définition stoïcienne du nom chez Apollonius et l'interprétation technique du passage en question par J. Lallot, il faut reconnaitre que l'on s'attendrait à voir mentionner la qualité générique (ou en l'occurrence, spéciale), plutôt que la substance générique. La présence de la notion de substance s'explique par le contexte, que nous avons déjà évoqué précédemment.

Comme on l'a vu, il s'agit, en ce début de la Syntaxe, de justifier la répartition des inquisitifs en deux parties du discours distinctes. Les interrogatifs comme "qui?", "quel?", "en quelle quantité?" portent sur le sujet ${ }^{38}$, sa substance ou ses accidents, et sont rangés parmi les noms. Les inquisitifs comme "comment?", "quand?", etc. portent sur le procès lui-même: ils concernent le signifié du verbe, et sont rangés parmi les adverbes. À cette division s'ajoute, pour la première classe d'inquisitifs, c'est-à-dire les noms, une bipartition substance/accident. Linterrogatif tis (qui?) porte sur la substance (Syntaxe II, § 32) et appelle une réponse nominale avec un appellatif ou un propre $(\$ 31)$, tandis que les autres questionnent sur les accidents (la qualité, la quantité, la taille, etc.) et appellent une réponse adjectivale (\$32). Le raisonnement semble attribuer aux propres et à certains appellatifs la signification de la substance et aux autres noms la signification des propriétés accidentelles comme la qualitée $e^{39}$, la quantité, la taille, etc. C'est la raison pour laquelle Apollonius parle ici

38 Même si ce texte pointe clairement vers une association entre diathèsis et verbe d'une part, et sujet (hupokeimenon) et nom, de l'autre, nous savons que nous n'avons pas pour autant affaire, avec le terme hupokeimenon, à une terminologie qui correspondrait clairement à un "sujet grammatical", notamment parce qu'il serait bien distinct du nom attribut. Ce sujet possède en effet aussi bien le sens d'un "référent" pour le nom, indépendamment de sa place dans la phrase, celle d'un sujet de l'institution du nom luimême, et, celle d'un substrat ontologique et physique pour la qualité qui le détermine. J. Lallot a souligné le fait que la notion de "sujet grammatical" était visée par toute une variété de termes, faisant l'hypothèse, avec D. Donnet, que cette approximation était liée au fait que la place sujet ou la place attribut d'un nom n'a pas de traduction morphologique (du fait de l'absence de "cas prédicatif"), voir Lallot (1997: I, p. 69-71). Il s'agit par exemple de la qualité d'être noble (voir Syntaxe I, 4). La qualité en question est à rapprocher d'un sens catégorial de "qualité", la 3eme catégorie accidentelle chez 
de "substance" en lieu et place de la qualité, qualité dont la signification est propre aux noms par oppositions aux pronoms: comme nous l'avons vu précédemment, la substance dont il s'agit ici est une sorte de qualité, une qualité substantielle précisément. La qualité signifiée par le nom correspond, selon le cas, à une substance/ essence, à une qualité, à une quantité, etc. Il s'agit donc d'une propriété en général, susceptible de contenir des traits essentiels ou accidentels.

Cette analyse permet de montrer comment chez Apollonius lui-même la substance peut être l'équivalent de la qualité.

Le même type d'hypothèse doit présider à l'analyse de la définition du nom rapportée par Choeroboscos ${ }^{40}$ :

Texte 12 (Choeroboscos: GG IV/1, p. 105, 23-5)

Le nom est une partie casuelle du discours qui assigne à chacun des sujets, corps ou choses, une substance commune ou particulière.

Cette définition implique l'existence d'un sens de "substance" qui soit l'équivalent d'une qualité, ou une substance seconde dans le vocabulaire aristotélicien, et non, évidemment, une substance-substrat. Cette dernière est exprimée dans la définition à l'aide de la notion de sujet (hukeimenon). Sachant que les "corps" et les "choses" mentionnés sont "subjectés", la substance commune ou particulière assignée aux référents doit revêtir un sens différent, irréductible à la substance-sujet (substrat) à laquelle elle est distribuée. On ne peut assigner un substrat à un substrat. Il doit s'agir, de nouveau, d'une propriété à laquelle il convient de donner un sens très élargi d'un point de vue ontologique, puisqu'elle doit englober le signifié de noms qui n'ont rien à voir avec une substance, qu'elle soit aristotélicienne ou stoïcienne, comme "blanc" ou "éducation", soit toute détermination en général ${ }^{41}$. Choeroboscos qui nous

Aristote, ou, comme le note F. Ildefonse (1997: p. 304) de la manière d'être stoöcienne, ce qui revient à la qualité au sens 2 selon $\mathrm{M}$. Baratin, à distinguer de la qualité comme signifié général des noms, la qualité au sens 1 selon M. Baratin. Que F. Ildefonse juge "apollonienne" (voir supra n. 36).

${ }^{41}$ Lanalyse que Choeroboscos propose de cette définition confirme ce point, car le "sujet" (hupokeimenon) est ce que les grammairiens appellent "les étants (onta) et les existants (huparchonta)", les corps étant ceux qui tombent sous les sens, dont un exemple est "homme". Or le même exemple, "homme", figure lorsqu'il s'agit de gloser la "substance commune", celle-ci étant "ce qui s'applique à une pluralité", comme l'homme ou le cheval (GG IV/1, p. 105, 29-106, 1). Il serait dépourvu de sens de dire que l'on assigne 
dit que Philopon et Romanos parlent de "qualité" à la place de "substance" dans la définition (ce qui est exactement contraire au témoignage des Scholies précédemment mentionné, voir supra texte 11). La manière dont il comprend cette variante est assez significative, et va dans le sens de la lecture proposée. Choeroboscos cite la définition de Romanos et de Philopon ("le nom est une partie casuelle du discours qui assigne à chacun de sujets, corps ou choses, une qualité commune ou particulière"), nous dit qu'ils mettent "qualité" à la place de "substance" et note: "puisque la qualité est également considérée dans la substance". "Il faut savoir", ajoute Choeroboscos, que "la substance est l' existence auto-subsistante (autupostatos huparxis) comme l'homme, le cheval et les autres semblables, la qualité le qualifié lui-même, comme le blanc, le blond, le noir et les autres semblables" (GG IV/1, p. 106, 7-10). Si tous les noms doivent assigner une "substance" à un sujet, suivant la définition attribuée à Apollonius par Choeroboscos, cette substance doit comprendre des qualités comme "blanc", à moins de n'inclure dans les noms que les noms désignant ce qui est ontologiquement une substance, comme "homme"; inversement si tous les noms doivent assigner une "qualité" à un sujet, suivant la définition attribuée à Apollonius par les Scholies et suivant Romanos et Philopon (selon Choeroboscos), cette qualité doit inclure la substance, afin de concerner aussi bien les noms signifiant l'existence auto-substistante que ceux signifiant le qualifié, à l'instar des noms épithètes.

\subsection{Le nom signifie ce que signifie le pronom, mais non l'inverse}

Un autre passage de la Syntaxe vient militer en faveur de la formulation tirée du Peri antônumias ("le nom [signifie] la substance avec la qualité") et conduit F. Ildefonse à considérer celle-ci comme une "précision" par rapport à la signification de la qualitét

\section{Texte 13 (Syntaxe I, §138)}

L'identité] substantielle (ousiôdes) est impliquée pour ceux qui sont nommés, mais pas l'institution propre des noms pour ceux qui subsistent. On peut

l'homme à l'homme, si ce qui est assigné et ce à quoi on assigne quelque chose ne sont pas différenciés. Il doit s'agit pour la première occurrence des sujets sensibles, la substance-sujet qui tombe sous le sens, et pour la seconde d'une essence qui leur est attribuée.

42 "Le nom, qui signifie la qualité, signifie plus précisément la substance avec la qualité" (Ildefonse, 1997: p. 309). 
être "celui-ci" sans être forcément "Ulysse", mais si on est "Ulysse", on est forcément du même coup "celui-ci"

Apollonius semble ainsi affirmer dans la Syntaxe que la signification du pronom est comme impliquée dans celle du nom (et non l'inverse). Il convient de revenir en détail sur le contexte argumentatif pour donner un sens précis à ce passage.

Apollonius traite alors des verbes signifiant l' "identité" (huparxis) ${ }^{44}$, dont on sait, par le paragraphe précédent (\$136), qu'elle peut être "nominale ou substantielle", ce qui correspond aux verbes comme "se nommer" (s'appeler) (onomazein) ou "être" (eimi). Ils peuvent être ajoutés à une construction qui a perdu sa congruence du fait que le verbe initial a été transformé en participe à cause de l'ajout d'un article devant l'accusatif. Il s'agit en effet d'énoncer une règle générale présidant à la transformation d'une phrase comme "Théon a insulté l'homme" en "Celui qui a insulté l'homme est / s'appelle Théon" (littéralement: "l'ayant insulté l'homme est / s'appelle Théon"). Pour qu'une phrase où l'on a rajouté un article devant l'accusatif (“*ho tòn ánthrôpon húbrisen Théôn [le (nomin.) l'homme (acc.) Théon (nomin.) a insulté]" ${ }^{\prime 5}$ redevienne congruente, il faut que le verbe initial devienne participe pour redonner à l'article "un [casuel] au cas droit et de genre masculin" ${ }^{46}$. Mais il est alors nécessaire de rajouter en outre un verbe pour que la seconde construction, à l'instar de la première, soit complète. On peut aussi transformer de façon analogue des phrases où un pronom figure en position de sujet, mais en ce cas, seul un des deux verbes d' "identité" est possible: le verbe "être".

43 GG II/2, p. 113, 11-13; Lallot, 1997: I, p. 141 (traduction modifiée).

44 Nous préférons conserver ici la même traduction pour huparxis que celle qui avait prévalu précédemment à propos de la double réponse (nominale et pronominale) possible pour une question avec le pronom "qui?" (voir supra Texte 2). J. Lallot traduit ici huparxis par "existence", mais il explique aussi que la notion visée ici par Apollonius est celle de copule: "cette formule met en évidence que les 'verbes d'existence' sont les verbe de type copule: il n'y a pas de verbe signifiant l' 'existence onomastique(?)', mais des verbes de type 's'appeler' qui introduisent un nom propre (onoma) comme prédicat; quant à l' 'existence substantielle', ici encore traduction littérale, elle ne renvoie à rien d'autre qu'à la prédication articulée par le verbe 'être”' (Lallot, 1997: II, p. 70, n. 291). La traduction de la notion d'huparxis par "identité", conforme à celle que nous avons suivie pour les textes sur l'inquisitif "qui”, permet d'indiquer l'existence d'une configuration conceptuelle commune aux deux cas.

45 I $~ 137 ;$ GG II/2, p. 112, 15; Lallot, 1997: I, p. 140.

46 I § 137; GG II/2, p.112, 17-113, 1; Lallot, 1997: I, p. 140. 
On ne peut passer de "celui-ci a insulté l'homme" qu'à "l'ayant insulté l'homme est celui-ci" (i. e. "celui qui a insulté l'homme est celui-ci"). La raison pour laquelle le pronom ne peut se construire qu'avec le verbe "être" est liée à la délimitation stricte de sa signification: C'est que les pronoms signifient seulement la substance qui est justement le signifié de "être" 4 .

En revanche les deux verbes sont possibles dans une construction avec un nom. C'est en justifiant cette double construction possible avec un nom qu'Apollonius affirme que l'identité substantielle est comprise dans le fait de recevoir un nom (être nommé, c'est être), mais non inversement (on peut être sans être nommé): c'est notre Texte 13.

Si les pronoms ne signifient que la substance, de sorte qu'ils ne peuvent admettre une construction "copulative" qu'avec le verbe "être", tandis que les noms impliquent aussi bien le fait d'être nommé que celui d'être, ou l'identité substantielle, faut-il en déduire que les noms signifient la substance, outre la qualité, voire que les noms signifient ce que signifient les pronoms (la substance) outre la qualité? La dernière formulation nous paraît inacceptable car la signification de la substance par les pronoms est une propriété de signification qui appartient en propre aux pronoms comme partie du discours distincte des noms. En aucun cas les noms ne peuvent s'approprier ou contenir ce qui fait la propriété de signification d'une autre partie du discours. Comment faut-il alors comprendre le fait que la substance est impliquée ou "présupposée", comme "prémisse préalable", selon la formule F. Ildefonse (1997: 302 sqq.), dans la signification des noms?

Il faut remarquer que l'argument d'Apollonius ne vise pas directement la signification des mots, noms et pronoms, mais ce qui est impliqué pour les réalités désignées par un nom ou un pronom. Il montre ce qui est impliqué, du côté des réalités, dans l'institution des noms. L'acte d'institution des noms implique qu'il y ait quelque chose pour recevoir ce nom: être nommé implique d'être; se voir attribuer un nom, c'est pouvoir être désigné par "ceci". Il ne suffit pas en revanche d'être pour recevoir un nom, alors que le simple fait d'être suffit à pouvoir être désigné par un pronom. La réalité substantielle (ousiôdes) impliquée par le nom relève de la description des conditions concrètes de l'institution d'un nom: pour donner un nom, il faut qu'il y ait quelque chose à nommer, un substrat de la nomination que l'on désigne par un pronom déictique. Elle est liée à la structure de la réalité nommée: si quelqu'un est désigné par le nom "Théon", il peut être désigné par le pronom

47 GG II/2, p. 113, 7-8; Lallot, 1997: I, p. 140. 
"celui-ci". La conception de l'imposition des noms rapportée par Apollonius va bien dans ce sens: on ne s'adresse ni au nouveau-né lui-même, ni à nous-mêmes, mais on le désigne à la troisième personne en donnant le nom, ce qui explique la raison pour laquelle le fait que les noms sont à la troisième personne relève d'une nécessité absolue $^{48}$. Cela ne veut pas dire que la signification de la substance, propre au pronom, est incluse dans la signification du nom. L'institution des noms n'implique donc pas que le nom possède la signification de la substance comme mode de signifier, mais elle implique qu'en attribuant un nom elle présuppose la réalité substantielle de ce qui est nommé, c'est-à-dire le sujet réel de la nomination.

Or la présupposition, dont F. Ildefonse a souligné la fécondité au sein de l'analyse linguistique d'Apollonius dans l'horizon d'une identification ou d'une détermination du référent ${ }^{49}$, nous semble se jouer sur le plan des choses signifiées, et non sur celui des propriétés de significations. Comme le formule F. Ildefonse dans un contexte différent, mais qui nous semble pertinent ici aussi, la présupposition n'indique pas nécessairement que ce qui est présupposé est signifié conjointement. Dans le même esprit, on peut dire que la signification de la qualité implique un substrat pour cette qualité, c'est-à-dire, d'une certaine manière, une "substance", et lire la définition du nom attribuée à Apollonius dans ce sens (Texte 11), où le sujet est compris comme une réalité sous-jacente aussi bien à la nomination qu'à la détermination de la qualité signifiée par le nom. Il faut donc distinguer la signification de la "substance" par le nom, qui désigne les conditions réelles dans lesquelles s'applique la nomination et la "détermination" du référent (l'existence d'une réalité subsistante à la base de l'acte d'imposition, d'une réalité qui soit substrat de la qualité signifiée) de la signification de la substance chez le pronom, laquelle relève de la description des modes de signifier et non des signifiés eux-mêmes.

\subsection{Le pronom signifie des qualités}

F. Ildefonse parle de "court-circuit" lorsqu'elle analyse le fait que le nom en vient à signifier aussi la substance (hupokeimenon, ousia) (Ildefonse, 1997: p. 308)

48 II, § 43; Lallot, 1997: I, p. 160.

49 Ce qui permet, selon elle, de dépasser le plan de la simple opposition "abstraite" entre nom et pronom en raison d'une opposition non moins abstraite entre substance et qualité (Ildefonse, 1997: p. 305 sqq.). La formule du Peri antônumias ("le nom signifie la substance avec la qualité”) est une pièce maîtresse de son analyse. Nous y revenons plus loin. 
dans notre Texte 2 par exemple (et ailleurs, comme nous l'avons vu), mais aussi pour comprendre le fait inverse, c'est-à-dire le fait que le pronom en vienne à signifier la qualité (à propos notamment du Texte 7) (Ildefonse, 1997: p. 322).

La même distinction, entre choses signifiées et propriétés de signification, vaut en effet à nos yeux pour la résolution du problème inverse, c'est-à-dire la signification de la qualité ou des "accidents" par les pronoms.

La Syntaxe paraît suggérer à plusieurs reprises que le pronom signifie des qualités: quand elle soutient que l'objet de la deixis pronominale est la qualité particulière, et non, comme on aurait pu s'y attendre, la substance du sujet (Texte 7), ou bien encore elle note que les accidents peuvent être également englobés dans la deixis (Texte 5). Nous rappelons les deux passages en question ${ }^{50}$ :

Il ressort clairement de là que, quand nous employons un nom [dans la question] nous nous enquérons de la substance (ousia) du sujet (hupokeimenon) : c'est elle et elle seule que désignent les pronoms (même si la deixis qu'ils opèrent oriente également vers les accidents (sumbébèkonta), d'où il suit qu'ils s'appliquent à n'importe quel sujet. Quand au contraire nous employons un pronom, c'est que nous avons déjà prise sur la substance, mais plus du tout sur la propriété (poiotès) qui s'y ajoute suivant l'institution du nom ${ }^{51}$.

De fait, potentiellement, c'est un nom propre qu'on donne à entendre quand on emploie un pronom, pas le nom sous sa forme phonétique, mais ce qui est l'objet de la deixis pronominale, à savoir la qualité propre du sujet ${ }^{52}$.

Sensible au problème que pose la signification de la seule substance comme propriété de signification du pronom liée à ses capacités déictiques, F. Ildefonse propose une méthode d'analyse qui est celle que nous avons utilisée pour tenter d'éclairer la question, inverse, de la signification de la substance par les noms, en distinguant le fait d'être visé conjointement et celui d'être signifié conjointement. Les pronoms signifient la substance par une deixis qui doit être comprise dans sa réalité physique. Quand on désigne du doigt une chose présente en chair et en os,

50 On peut également mentionner Syntaxe II, 24, comme le rappelle F. Ildefonse (1997: p. 308).

51 GG II/2, p. 101, 11-102, 3; Lallot, 1997: I, p. 135.

52 GG II/2, p. 155, 3-5; Lallot, 1997: I, p. 158. 
les accidents ou les qualités sensibles de cette chose sont nécessairement associés à la deixis, sans en être pour autant l'objet:

Le pronom, qui signifie l'existence ${ }^{53}$ ou la réalité du référent, visant ce référent qui est un objet déterminé [...], remplace le nom propre. La situation sensible de la deixis, lorsque son protocole est optimal, lui fait envelopper les accidents de cet objet singulier, qui sont nécessairement visés conjointement [...] sans être pour autant signifiés conjointement (Ildefonse, 1997: p. 308-309; nous soulignons).

Lanalyse de J. Lallot va dans le même sens: les accidents enrichissent la deixis pronominale comme un "commentaire" (Lallot, 1997: II, p. 263, n. 64).

Il faut donc comprendre dans la désignation de la substance et de la qualité deux notions bien distinctes. La première exprime un mode de signification bien précis, celui-là même qui préside à la définition de la notion propre de la partie du discours à laquelle le mot appartient. En ce sens la propriété de signification du pronom est de signifier la substance et elle seule, car il identifie le référent en montrant sa réalité en chair en os, ici et maintenant, sa substantifique substance, la "présence de quelque chose de réel" (Ildefonse, 1997: p. 306). Il peut en outre signifier les qualités de ce référent, mais alors la signification de la qualité ne désigne pas un mode de signification: elle s'attache à la description de ce qui est signifié, en l'occurrence une réalité individuelle dotée d'un certain nombre de qualités sensibles, ce que F. Ildefonse décrit comme le "débordement de la deixis", celle-ci étant une "trouée sur le monde sensible" (Ildefonse, 1997: p. 308). On peut appliquer le même raisonnement à la signification de la substance par le nom, avec les nuances qui s'imposent. Le pronom peut bien signifier une qualité, puisqu'il n'y a pas de substance sans qualité, de même que le nom peut signifier une substance parce qu'il n'y a pas de qualité sans substance pour la porter, mais ces deux éléments appartiennent à la description des choses signifiées, et non à celle des modes de signifier.

\subsection{Le substrat comme signifié associé du nom ? Un troisième sens de "substance" ?}

Peut-on véritablement mettre la signification de la qualité par les pronoms et la signification de la substance par les noms sur le même plan?

53 Lauteur a précisé dans la note 2 de la page précédente qu'elle préférait traduire ici ousia par existence, plutôt que par substance. 
Pour la signification des noms, la distinction entre choses signifiées et propriétés de signification revient à dissocier le mode de signifier des noms, qui implique la seule qualité, et la nature de ce que les noms signifient, qui présuppose quelque chose de substantiel. De ce point de vue, les noms signifient toujours une substance et une qualité dans le sens où un substrat de la qualité et de l'institution du nom est toujours associé au nom. Cette approche nous semble de nature à expliquer comment la Syntaxe peut affirmer à la fois que le nom signifie la qualité, que les pronoms perdent leurs propriétés référentielles en l'absence de la chose tandis que, a contrario, les noms conservent leur capacité à identifier un individu en l'absence de ce dernier (voir Syntaxe II, § 42; supra n. 14), et que la réalité substantielle est impliquée pour ceux qui sont nommés (mais non l'inverse). Le mode de signification propre des noms est de signifier la qualité; en aucun cas ils ne signifient la substance comme le font les pronoms, c'est-à-dire identifient leurs référents en désignant directement les choses dans leur réalité (leur substance). Mais signifiant une qualité, ils désignent les substrats (hupokeimenon) qui possèdent cette qualité, substrats dont l'existence substantielle (ousiôdes) doit être présupposée pour l'institution des noms sans être pourtant nécessaire à l'accomplissement de la signification du nom (puisque les noms peuvent se référer à eux in absentia, contrairement aux pronoms). La signification des noms peut donc "présupposer" la réalité substantielle du sujet de la qualité et de la nomination, sans que la présence de ce sujet ne soit requise pour que s'effectue la désignation, contrairement au cas du pronom, qui a besoin de la présence de la chose - ou de celle d'un nom propre qui s'y substitue, et auquel il se réfère, comme dans le cas des échanges épistolaires.

On pourrait cependant introduire une nuance dans l'analyse des noms par rapport à celle des pronoms; les noms pourraient bien avoir le substrat comme signifié associé sémantiquement et non pas seulement ontologiquement. La définition du nom rapportée par les Scholies, si elle est bien apollonienne, donne au nom deux corrélats sémantiques, la qualité et le sujet qui la porte et auquel le nom attribue la qualité, tandis qu'il n'y en a qu'un seul pour le pronom. Les qualités éventuellement signifiées par les pronoms appartiennent sans équivoque à des caractéristiques nonlinguistiques des signifiés, et non à la signification elle-même. On pourrait lire cette définition de telle sorte que le substrat ne serait pas seulement ontologiquement présupposé (puisqu'il faut bien que la qualité signifiée ait un porteur ) - à l'instar des qualités, associée à la deixis pronominale parce que, de fait, aucune substance pure ne se présente à la désignation dépourvue d'accidents - mais bien sémantiquement présupposé. Le nom ne signifie pas seulement une qualité qui se trouve avoir besoin, sur un plan ontologique d'un substrat d'inhérence, ou d'un sujet à quoi être 
distribuée, mais le sujet est bien sur quoi le nom opère directement sa détermination, et qu'il nomme en lui distribuant une qualité. Il nous semble donc possible de retenir la nuance qui consiste à intégrer dans la signification du nom deux corrélats sans pour autant admettre parmi ceux-ci la substance signifiée par le pronom. Entre le point de vue de la propriété de signification et celui des présuppositions strictement associées aux caractéristiques ontologiques des choses signifiées, il serait possible de concevoir la référence au substrat de la qualité et de la nomination comme appartenant à la signification du nom, sans toutefois faire partie de la propriété de signification, ce qui explique le fait que les noms peuvent exercer leur nomination en l'absence du sujet, ce que les pronoms ne peuvent accomplir.

Introduit-on, avec cette nuance, un troisième sens de "substance" dans l'analyse de la signification des noms, la substance-sujet ou la "substance" au sens 1 ? Notons que le substrat de la nomination et de la qualité est généralement désigné par le vocable d'hupokeimenon, et non par celui d'ousia. L'hégémonie du terme "substantia" dans l'analyse des mêmes phénomènes chez Priscien a conduit à proposer l'existence trois sens de "substance", un sens de substance-sujet qui recouvre l'hupokeimenon apollonien (lui-même rendu par le subjectum chez Priscien), un sens de substance comme qualité substantielle, i. e. comme substance au sens 2 dans la classification de M. Baratin, la qualité substantielle ou la substance comme "essence", "substance seconde", et un sens de "substance" propre au pronom, qui décrit un mode de signifier spécifique. Si une notion de substance-substrat est probablement présente chez Apollonius, le vocabulaire de l'ousia ne fait pas à proprement parler partie de la description de la propriété de signification du nom lorsqu'il s'agit de décrire ce substrat. Le vocabulaire de l'ousia n'est pas complètement absent, avec la notion d'identité substantielle (ousiôdès) mais, comme on l'a vu, elle apparaît dans un contexte qui ne décrit pas systématiquement une propriété de signification, mais une présupposition d'existence du côté de ce qui est signifié par le nom.

Le dernier élément textuel généralement apporté au dossier de la signification de la substance, outre la qualité, par le nom pose un tout autre problème. L'ousia comme substrat de la qualité apparaît bien de manière explicite dans l'expression ousia meta poiotètos employée par le Peri antônumias à propos de la signification du nom. Or il s'agit d'une discussion qui porte clairement sur les propriétés de signification des parties du discours que sont le nom et le pronom. Cette formule est destinée à rendre compte de la signification générale des noms par opposition à celle des pronoms, et elle représente à ce titre une objection de taille aux analyses que nous venons de proposer. Elle introduit clairement la substance-substrat, la substance au sens 1 , non seulement comme signifié associé, mais comme partie de 
la propriété de signification du nom, du moins selon la lecture généralement proposé de ce passage. Une mise en contexte précise de cette formule pourrait cependant permettre d'en infléchir l'interprétation couramment admise.

\section{5. la signification de la substance avec la qualité dans le Peri antônumias et les trois sens de "substance" chez Apollonius}

Le passage du Peri antônumias qui attribue au nom de la signification de substance avec la qualité traite bien les modes de signifié du nom et du pronom (et non des éventuelles caractéristiques ontologiques de leurs signifiés réels). C'est sans doute la raison pour laquelle tous les spécialistes qui ont commenté la définition du nom chez le (Pseudo-)Denys le Thrace, Apollonius, ou Priscien (à commencer par l'éditeur des fragments des œuvres perdues d'Apollonius) ont considéré que la formule du Peri antônumias pouvait être mise sur le même plan que la formulation du propre du nom chez Priscien, ou bien encore sur le même plan que de la définition du nom propre et appellatif dans la Technè (voir supra n. 3, 36, 42, 49). Elle reflèterait ainsi la position d'Apollonius sur la définition du nom et sur sa propriété de signification, en l'absence d'œuvre conservée spécifiquement consacrée à ce sujet. Le contexte d'une discussion technique sur la possible confusion entre deux parties du discours, en l'occurrence le nom et le pronom à propos de la particule interrogative tís (qui?), où intervient la question des enclitiques, des terminaisons de déclinaisons etc., indique que nous avons affaire à des notions grammaticales bien délimitées. Comment comprendre cette formule si l'on sait que la signification de la substance par le nom ne peut en aucun cas être assimilée à la possession d'une deixis, comme c'est le cas pour le pronom où ces deux propriétés sont étroitement associées? Nous avons vu que la signification de la qualité et l'absence de deixis étaient deux traits connexes pour le nom, tandis que le fait de signifier la substance et celui de posséder une deixis étaient deux propriétés sémantiques étroitement liées dans la discussion sur l'utilité respective des pronoms et des noms (voir supra n. 3).

Il convient d'abord de rappeler le contexte précis dans lequel cette définition apparait. Ce contexte est essentiellement polémique, raison pour laquelle la capacité de l'énoncé "le nom signifie la substance avec la qualité" à valoir comme définition générale du propre du nom chez Apollonius doit être remise en question.

Nous avons vu qu'il s'agissait d'un passage où le grammairien traitait du problème des mots classés indûment dans l'une ou l'autre catégorie, les noms et les pronoms. Il donne alors la parole à des grammairiens qui soutiennent une position adverse, selon laquelle l'expression tís devrait être considérée comme un pronom (et 
non comme un nom, selon la doctrine d'Apollonius). Laffirmation que le pronom signifie "la seule substance" et le nom "la substance avec la qualité" est en réalité l'un des arguments qu'Apollonius met dans la bouche des autres grammairiens. Le raisonnement consiste à énoncer le principe, c'est-à-dire que le nom signifie la substance avec la qualité, puis à s'appuyer sur le fait que l'expression tís ne dévoile que la substance, pour affirmer ensuite qu'elle doit alors se ranger parmi les pronoms, conclusion qu'Apollonius récuse totalement. Mais admet-il les prémisses de ses adversaires (auquel cas il serait d'accord pour dire que le nom signifie la substance avec la qualité) dans un raisonnement qu'il considère comme faux par ailleurs, ou refuse-t-il l'argument du fait de la fausseté de ses prémisses?

Dans l'ensemble du passage concerné, les réponses d'Apollonius aux objections ne portent pas seulement sur de mauvaises conséquences tirées de maximes justes, mais elles visent également les principes sur lesquels on prétend se fonder. On le voit dans la réfutation du premier argument, qui affirme que tís est un pronom puisqu'il est enclitique: Apollonius précise bien que le fait d'être enclitique n'est pas propre au pronom, mais concerne aussi les verbes. Rien ne nous autorise donc à penser que les principes sur lesquels reposent les objections reflètent fidèlement la doctrine d'Apollonius et sont simplement mal interprétés ou mal utilisés, et qu'ainsi l'auteur de la Syntaxe adhèrerait à la prémisse citée par ses adversaires. La réponse du grammairien nous indique même le contraire. L'argument complet qu'il veut réfuter est le suivant: "Les pronoms signifient la substance, les noms la substance avec la qualité, donc tís (qui?), qui exprime seulement la substance, est un pronom" (GG II/1, p. 27, 9-10). La réponse d'Apollonius, une page plus loin, s'énonce ainsi:

\section{Texte 14 (Peri antônumias)}

Les inquisitifs [portent] sur la quantité, comme pósos (en quelle quantité?), sur la qualité comme poîos (quel?), sur la grandeur comme pèlikos (de quelle grandeur?), ou sur la substance (ousia) comme tís (qui?), mais [ce dernier] n'est pas pour cela un pronom (GG II/1, p. 28, 6-9).

Apollonius a fait clairement savoir précédemment que les autres interrogatifs étaient des noms, car étant indéterminés (aorista), ils sont le contraire d'un pronom, qui signifient des personnes déterminées (GG II/1, p. 27, 13-16). Le caractère laconique du passage nous oblige à combler l'ellipse en renvoyant à l'argumentaire précédemment étudié sur la répartition des inquisitifs dans la Syntaxe. Parmi les 
inquisitifs nominaux, ceux cités ici, nous avons vu qu'il convenait de distinguer l'inquisitif de substance (qui?) de ceux qui portent sur les accidents - la quantité, la taille, etc.. Dans ce même passage, on a trouvé l'affirmation selon laquelle le nom propre signifiait aussi la "substance générique", ce qui nous a conduit à expliquer dans quelles conditions un nom pouvait signifier la "substance" à titre de qualité, c'est-à-dire signifier une qualité qui se trouve être une substance (et non un accident).

On peut raisonnablement supposer qu'Apollonius renvoie implicitement au type d'arguments développés dans la partie introductive de la Syntaxe sur la répartition des inquisitifs nominaux ${ }^{54}$. Étant donné la prégnance de l'affirmation apollonienne selon laquelle le pronom signifie la substance dans le Peri Antônumias, il faut nécessairement, quand le même texte affirme que ce n'est pas parce qu'un inquisitif porte sur la substance qu'il est un pronom, que la notion de substance soit sensiblement différente. Comme nous l'avons vu, la particule ne peut être un nom que si elle signifie une qualité, c'est-à-dire si elle désigne son référent à travers l'attribution d'une détermination quelconque. L'argument cité par Apollonius présente fait apparaître une question pendante pour sa doctrine, puis pour la grammaire de Priscien, celle de la qualité signifiée par une particule comme tís, qui porte sur la substance en général, et peut recevoir en réponse n’importe quel nom propre, appellatif (pourvu qu'il ne signifie pas les accidents du sujet) ou pronom ${ }^{55}$.

54 Nous disons "le type d'arguments" et non "les arguments" car le Peri Antônumias ne saurait renvoyer implicitement à la Syntaxe, puisque les spécialistes s'accordent pour penser que les traités sur les différentes parties du discours ont probablement été rédigés avant la Syntaxe, œuvre de synthèse (Lallot, 1997: I, p. 32-33, n. 56).

55 C'est précisément de problème qui conduira en partie Priscien à construire la notion de "noms généraux" eux-mêmes au-dessus, dans leur généralité, des noms génériques comme "animal". À la question de la "qualité" signifiée par le nom "qui?», on peut ainsi répondre qu'il s'agit de la qualité d'être une substance tout court, par différence avec un nom comme "homme", ou "animal". Il faut dire que Priscien est confronté à la nécessité de faire entrer dans la catégorie des noms l'équivalent des articles post-positifs, comme qui, qu'Apollonius rangeait dans les articles, inexistants en latin, ce qui donne à la question de la qualité signifiée par les noms indéfinis une acuité encore plus grande. Léquivalent des inquisitifs nominaux et des articles post-positifs grecs (comme quis/qui, qualis, quantus ...) est désigné par la notion de "nom général". Ces noms signifient chaque type de détermination que peut prendre la qualité 1 en général (c'est-à-dire la substance 2, la qualité 2, la quantité, le nombre): ainsi certains signifient la substance en général, d'autres la qualité en général etc. (Baratin, 1989: p. 405-407; Rosier; Stefanini, 1990, p. 285-303). 
On peut en conclure que l'argument des adversaires d'Apollonius prétend bien situer la discussion sur les modes de signifier, ceux-là même qui permettent de situer un mot dans l'une ou l'autre partie du discours. Mais ces grammairiens classent indûment un mot parmi les pronoms au lieu des noms parce qu'ils confondent justement la propriété de signification et la description de la nature des signifiés. Ainsi, parce que ce que signifie l'inquisitif tís est une substance, et même seulement une substance (c'est-à-dire aucun accident du substrat tel que sa qualité, sa taille, etc.), ils attribuent à cette expression la substance comme propriété de signification, et le considère comme un pronom. Mais, comme le souligne Apollonius en quelques mots, l'un n'entraîne pas l'autre.

\section{Conclusion}

Sans nier une certaine pertinence à l'idée que le nom puisse signifier chez Apollonius "la substance avec la qualité", au sens où le substrat est "présupposé" non seulement ontologiquement, mais aussi sémantiquement comme signifié conjoint dans la signification du nom, plusieurs éléments viennent mettre en question l'adhésion du grammairien grec à la formule du Peri antônumias si l'on doit y voir la description du mode de signification du nom. Elle ne correspond pas aux formules habituelles de la Syntaxe, ni au cœur de sa doctrine sur le rapport de complémentarité entre nom et pronom au livre II, qui est censé être postérieur au Peri antônumias, et constituer une synthèse de l'enseignement d'Apollonius. Nous avons en outre vu que cette formule était mise par Apollonius dans la bouche de ses adversaires, et que l'argument était réfuté dans son ensemble dans la page suivante du traité. Le fondement de la critique d'Apollonius, qui consiste à souligner que ce n'est pas parce qu'un mot porte sur la seule substance qu'il a la signification de la seule substance comme notion propre ou propriété de signification, laisse entendre que la formule: "la substance avec la qualité" pourrait elle-même relever d'une confusion entre le point de vue des signifiés et celui du mode de signification. On érigerait en propriété de signification ce qui est seulement un trait du signifié.

Même si l'on devait supposer qu'Apollonius adhère à la prémisse posée par ses adversaires ("le nom signifie la substance avec la qualité") dans raisonnement faux par ailleurs - hypothèse qui reste possible, quoique peu plausible - et l'admettre ainsi aux côtés de la signification de la seule qualité, majoritaire dans la Syntaxe, la substance en question ne saurait être celle qui constitue le mode de signifier du pronom. Apollonius manierait alors de manière implicite non seulement les deux sens de "substance" comme qualité substantielle et comme propriété de signification attachée en propre aux pronoms, mais aussi un troisième sens de "substance", la substance-substrat. 


\section{RÉFÉRENCES BIBLIOGRAPHIQUES}

BARATin, M. "À propos du nom propre dans l'Antiquité: quelques points qui ont fait débat”, Corpus 50, 2006, p. 229-237.

- "Sur les notions de sujet et de prédicat dans les textes latins", Archives et documents de la Société d'histoire et d'épistémologie des sciences du langage 10/2, 1994, p. 49-79.

. La Naissance de la Syntaxe à Rome, Paris, 1989.

BARnes, J. Porphyry, Introduction, Oxford, 2003, p. XIX.

Blank, D. Ancient philosophy and grammar. The Syntax of Apollonius Dyscolus, 1982.

Brumberg-Chaumont, J. "Grammaire et logique du nom d'après les Gloses sur Priscien de Guillaume de Conches". In: Guillaume de Conches: Philosophie et science au XIle siècle, éd. I. Caiazzo et B. Obrist, Firenze, 2011, p. 377-465.

. "La signification de la substance chez Priscien et Pierre Hélie". In: Priscien, éd.

L. Holtz, M. Baratin, et B. Colombat, Turnhout, 2009, p. 503-519.

Brunschwig, J. Études sur les philosophies hellénistiques, Paris, PUF, 1995.

. "Remarques sur la théorie stoïcienne du nom propre", Histoire, Épistémologie, Langage 6, 1984, p. 3-19.

Chiaradonna, R. "What is Porphyry's Isagoge?", Documenti e Studi sulla Tradizione Filosofica Medievale XIX, 2008, p. 1-30.

. "La teoria dell'individuo in Porfirio e l' idiôs poion stoico", Elenchos, XXI/2, 2000, p. 303-331.

EbBesen, S. "Priscian and the Philosophers". In: Priscien: Transmission et refondation de la grammaire de l'Antiquité aux Modernes, éd.. L. Holtz; M. Baratin; B. Colombat, Turnhout, 2009, p. 109-124.

. "The Traditions of Ancient Logic-cum-Grammar in the Middle Ages - what's the problem?". In: The Many Roots of Medieval Logic: the Aristelian and the NonAristotelian Traditions, éd. J. Marenbon, Vivarium 45, 2007 (numéro spécial), p. 136-152.

Garcea, A. "Substance et accidents dans la grammaire de Priscien". In: Priscien: Transmission et refondation de la grammaire de l'Antiquité aux Modernes, éd.. L. Holtz; M. Baratin; B. Colombat, Turnhout, 2009, p. 125-138.

Goulet, R. "La classification stoïcienne des propositions simples selon Diogène Laërce VII 69-70". In: Les Stoïciens et leur logique, éd. J. Brunschwig, Paris, 1978, p. $171-198$ 
Gourinat, J.-B. La Dialectique des Stoïciens, Paris, 2000.

ILDEFONSE, F. "Remarques sur la théorie stoïcienne de la phrase (énoncé, proposition) et son influence chez les grammairiens". In: Théories de la phrase et de la proposition, éd. P. Büttgen, S. Diebler et M. Rashed, Paris, 1999, p. 151-170. . La Naissance de la Grammaire dans l'Antiquité grecque, Paris, 1997.

LALLOT, J. "Les philosophes des grammairiens, les allusions aux philosophes dans les textes grammaticaux de la tradition alexandrine". In: Actualité des Anciens sur la théorie du langage, éd. R. Petrilli et D. Gambarara, Münster, 2004, p. 111-127. . La Grammaire de Denys le Thrace, Paris, 1998 (2 ${ }^{\text {nde }}$ édition). . Apollonius Dyscole. De la construction, Paris, 1997.

. "Origines et développement de la théorie grammaticale des parties du discours", Langages 92, 1988, p. 11-23.

Long, A.; Sedley, D. Les Philosophes hellénistiques II, Les Stoïciens, trad. J. Brunschwig et P. Pelegrin, Paris, 2001.

Luhtala, A. Grammar and Philosophy in Late Antiquity, Amsterdam, 2005.

LunA, C. Commentaire sur les Catégories d'Aristote, Paris, 2001.

Rosier, I.; Stefanini, J. "Théories médévales du pronom et du nom général”. In: De Ortu Grammaticae, Studies in the History of the Language Sciences, 43, éd. G. L. Bursill-Hall, S. Ebbesen et K. Koerner, Amsterdam, 1990, p. 285-303.

SeXtus EmpiRIcus, Against the Logicians II, trad. anglaise et texte grec R. G. Bury, Londres, 1967.

Sluiter, I. Ancient grammar in Context, Amsterdam, 1990.

Wouters, A. "Plutarch's Comments on Plato's 'grammatical' (?) Theories". In: Plutarchea Lovaniensia, A Miscellany of Essays on Plutarch, éd. L. Van Der Stockt, Louvain, 1996, p. 309-328.

J. Brumberg-Chaumont. Os sentidos de "substância" em Apolônio Díscolo.

RESUMO: $O$ artigo dedica um exame detalhado aos sentidos de substância presentes em Apolônio Díscolo. A primeira dificuldade é de ordem metodológica, pois é necessário examinar uma noção 
eminentemente filosófica no seio de um texto gramatical da Antigüidade tardia. É necessário, pois, precisar qual pode ser o referente filosófico a que se pode associar o uso que Apolônio faz da noção de substância, levando-se em conta a forte influência de noções estóicas e ainda de uma gama de influências peripatéticas e também médio-platônicas e neo-platônicas. A essa dificuldade metodológica soma-se o obstáculo de um corpus lacunar, a que falta a parte relativa ao nome. É necessário, pois, reconstituir a doutrina apoloniana a partir do que se encontra, de maneira esparsa, na Sintaxe e no Acerca do pronome. Então, muitos elementos permitem questionar a adesão de Apolônio à fórmula do Acerca do pronome, a qual não corresponde às fórmulas habituais da Sintaxe, nem ao coração da doutrina sobre a relação de complementaridade entre nome e pronome do "Livro II" desta, o qual se admite ser posterior ao Acerca do pronome e constituir uma síntese do ensinamento do gramático grego. Apolônio manusearia, então, de maneira implícita, não só os dois sentidos de substância como qualidade substancial e como propriedade de significação vinculada de modo próprio ao pronome, mas também um terceiro sentido de substância, o de substância-substrato.

PALAVRAS-CHAVE: Apolônio Díscolo, Prisciano; ousía, hýparxis, hypokeímenon, deîxis; substantia, subjectum; substância, qualidade, quantidade; nome, adjetivo, pronome. 